Ann. Biol. anim., Bioch., Biophys., Ig66, 6 (2), 223-248.

\title{
RECHERCHES GÉNETIQUES SUR L'HÉMIMÉLIE LONGITUDINALE CHEZ LA SOURIS. ÉTUDE DE LA PÉNÉTRANCE
}

\author{
N. KOBOZIEFF, Nathalie POMRIASKINSKY-KOBOZIEFF, \\ E. GEMÄHLING et F. REYNES \\ Laboratoire de Génétique, \\ École nationale vétérinaire d'Alfort, 94 -Maisons-Alfort
}

\section{SOMMAIRE}

Cette étude génétique sur l'hémimélie longitudinale a porté sur 8801 souris, appartenant à quatre milieux génétiques différents : $336 \mathrm{I}$, RAP ; $3689 \mathrm{MO}$; I I $74 \mathrm{C}_{3} \mathrm{H}$ et $577 \mathrm{C}_{57} \mathrm{BL}$.

Pour chacun de ces milieux génétiques nous avons effectué quatre principales séries de croisements groupant de neuf à douze types d'expériences.

Les souris hémiméliques se comportent comme des homozygotes récessifs de constitution génotypique he/he, la pénétrance est absolue.

Les hétérozygotes de constitution génotypique + /he sont à phénotype exprimé (polydactyle) ou à phénotype non exprimé (normal overlaps) de ce fait la pénétrance n'est pas absolue.

La pénétrance de la polydactylie varie en fonction du milieu génétique. Elle est forte pour les milieux $\mathrm{RAP}$ et $\mathrm{C}_{57} \mathrm{BI}$, et plus faible pour $\mathrm{C}_{3} \mathrm{H}$ et $\mathrm{MO}$, surtout pour ce dernier.

On relève en outre une variation de la pénétrance à l'intérieur de chacun des milieux génétiques; elle dépend de différents facteurs :

du degré d'expressivité de la polydactylie chez les géniteurs utilisés et de leur sexe;

du géniteur normal ;

du mode de croisement : la pénétrance est plus faible dans la première génération que dans la descendance issue de croisements de retour.

\section{INTRODUCTION}

Nous avons publié précédemment une étude morphologique détaillée de l'hémimélie longitudinale chez la souris, (symbole he) ainsi que l'étude génétique préliminaire de cette anomalie. L'étude génétique détaillée, mode exact de transmission, 
pénétrance, expressivité, influence du milieu génétique des lignées normales introduit dans le patrimoine des mutants, fera l'objet de cet article.

Les anomalies héréditaires du squelette des membres présentent une grande diversité phénotypique et se rencontrent dans les différentes espèces, tant chez les animaux de laboratoire : souris, rats, cobayes que chez les animaux domestiques: chats, chiens, élevages de volailles, chèvres, moutons, porcs, bovins et chevaux. On rencontre également des anomalies semblables chez l'homme. Elles intéressent le segment distal (ou acromélique) seul : orteils, métatarse, tarse pour les membres postérieurs ; doigts, métacarpe et carpe pour les membres antérieurs, ce sont alors l'hyperphalangie du doigt I, l'oligodactylie, la syndactylie et la polydactylie. L'anomalie atteint aussi le segment moyen (mésomélique), on observe alors des hémimélies (ou péromélies selon les auteurs allemands), radiales ou cubitales pour les membres antérieurs ou hémimélies tibiales ou péronéales pour les membres postérieurs ; enfin, l'anomalie s'étend parfois même au segment proximal (rhizomélique), elle est associée ou non aux anomalies du segment distal ou à d'autres anomalies de squelette axial notamment.

\section{MATÉRIEI, E'T 'TECHNIQUES}

En I946, dans un stock de souris à oreilles tronquées, nous avons vu apparaitre trois formes d'anonalie podale : pied-bot, contracture des membres et polydactylie; les sujets atteints étant peu viables, cette anomalie a disparu. Cependant, à partir des souris normales de ce stock, par un inbreeding intense, nous avons abouti à la création de la lignée L (souris très légères). Celle-ci a servi à une étude pondérale pour laçuelle nous avons procédé à des croisements avec des souris particulièrement lourdes appartenant à la lignée $\mathrm{P}$ (lignée constituée à partir de souris très lourdes qui nous avaient été confiées en 1943 par le 10r PainTEAL, et qui n'avaient jamais fourni aucune mutation).

C'est à la suite des croisements effectués entre ces deux lignées qu'en I949 nous avons vu apparaître à nouveau des anomalics podales se présentant sous diverses formes. Anomalies légères, intéressant le segment distal des membres postérieurs seuls (hyperphalangie, polydactylie). Anomalies graves, portant sur les trois segments des membres postérieurs, se traduisant par une hémimélie tibiale accompagnée d'hyperphalangie du doigt $\mathbf{I}$, de polydactylie ou d'oligodactylie; parfois accompagnées de polydactylie ou d'hyperphalangie du doigt I des membres antérieurs et de raccourcissement de la queue avec coudures.

Dans une note précédente, portant sur l'hémimélie longitudinale chez la souris, nous avons démontré que la pénétrance des hétérozygotes, quel que soit le type de croisement utilisé, est non absolue, contrairement à ce qui a été observé pour les homozygotes.

Au point de vue statistique nous avons utilisé le test $\chi^{2}$ de Kard Pearson que ce soit comme test d'ajustement, comme test d'homogénéité, ou comme test d'indépendance de deux caractères (table de contingence).

Tous avons maintenant cherché à connaître quel était le rôle exact du milieu génétique des différentes lignées normales introduit dans les croisements au cours de nos recherches.

Il nous est apparu, en effet, que le milieu génétique des géniteurs normaux introduit dans le patrimoine héréditaire des sujets mutants, a une action sur la pénétrance et l'expressivité des hétérozygotes et sur l'expressivité seule des homozygotes. Nous avons d'abord étudié les effets observés sur la pénétrance.

Pour cette étude nous avons pris en considération quatre lignées normales : une lignée française $(\mathrm{MO} / \mathrm{Ko})$ et trois lignées d'origine américaine ( $\mathrm{RAP} / \mathrm{Ko}, \mathrm{C}_{3} \mathrm{H} / \mathrm{Jax}-\mathrm{Ko}_{0}$ et $\mathrm{C}_{57} \mathrm{BL} 6 / \mathrm{Ko}$ ) ${ }^{(1)}$, entretenues depuis longtemps dans notre laboratoire, bien connues du point de vue héréditaire et qui étaient e mieux représentées dans les différents types de croisements.

(1) Pour plus de commodité, dans la suite de cet article, nous désignerons les lignées par: MO, RAP, $\mathrm{C}_{3} \mathrm{H}$ et $\mathrm{C}_{57} \mathrm{BL}$ 
Pour des raisons matérielles. il n'a pas toujours été possible d'effectuer tous les types de croisements dans chacun des milieux génétiques.

Nous avons effectué deux groupes de croisements :

\title{
Croisements fondamentaux.
}

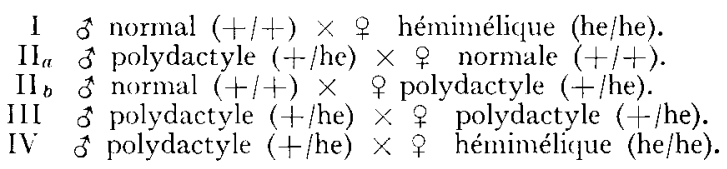

\section{Croisements permettant l'analyse de la descendance d'apparence normale}

\author{
$V$ o Nov issu du $\mathrm{I}(+/ \mathrm{he}) \times q$ hémimélique (he $/$ he). (Non $=$ normal overlaps). \\ VI ot polydactyle $(+/$ he $) \times$ o $F_{1}$ Nov du I $(+/$ he $)$. \\ VII of $\mathrm{F}_{1}$ Nov du I $(+/$ he $) \times{ }^{+} \mathrm{F}_{\mathrm{I}}$ Nov du I $(+/$ he $)$. \\ VIII $_{a}$ of polydactyle ( $+/$ he $) \times q \mathbf{F}_{\mathrm{l}}$ Nov du II ( + /he). \\ VIII $_{b}$ of polydactyle $(+/$ he $) \times \frac{9}{F_{1}}$ normale du II $(+/+)$. \\ IX $_{a}$ o $\mathrm{F}_{1}$ Nor du II $(+/$ he $) \times$ o $\mathrm{F}_{1}$ Nor du II ( $\left.+/ \mathrm{he}\right)$. \\ $\mathrm{IX}_{b}$ o $\mathrm{F}_{1}$ Nor du II $(+/$ he $) \times$ o $\mathrm{F}_{1}$ normale du II $(+/+)$. \\ IX. a $F_{1}$ normal du II $(+1+) \times \frac{}{+} \mathrm{F}_{1}$ nommale du II $(+1+)$.
}

Ceci nous a permis d'étudier le comportement génétique des souris polydactyles ou atteintes d'hénimílie.

Étant donné (que la pénétrance n'est pas abolue chez les hétérozygotes, nous avons jugé utile d'analyser la descendance d'apparence normale qui est en excédent et dépisté les sujets normal overlaps, qui se comportent comme des hétérozygotes polydactyles. Aous avons comparé la pénétrance des homozygotes et des hétérozygotes issus de croisements avec des sujets des quatre lignées normales utilisées dans cette expérience.

Nous avons aussi étudié et comparé l'influence des géniteurs homozygotes et hétérozygotes sur la pénétrance chez les descendants issus de leur croisement avec des sujets de lignées normales et ceci pour chacune des lignées normales utilisées. cendants.

Enfin, nous avons étudié la distribution des sexes dans les différentes classes de des-

\section{RÉSULTATS}

Au cours de l'étude génétique préliminaire, les résultats des croisements entre souris polydactyles ou hémiméliques entre elles ou avec des souris normales, ont montré que les souris polydactyles sont hétérozygotes, donc de constitution génotypique $(+/$ he) et les souris hémiméliques-homozygotes (he/he).

Ceci nous a conduits à émettre l'hypothèse que les quatre séries de croisements $\left({ }^{1}\right)$ d'hémiméliques (he/he) et de polydactyles $(+/$ he) devraient donner les résultats suivants :

\footnotetext{
I normal $(+/+) \times$ hémimélique (he/he) $\ldots . .(+/$ he $)$, tous polydactyles ;

II polydactyle $(+/$ he $) \times$ normal $(+/+) \ldots \ldots$ I $(+/$ he $):$ I $(+/+)$;

III polydactyle $(+/$ he $) \times$ polydactyle $(+/$ he $) \ldots \mathrm{I}(+/+): 2(+/$ he $):$ I (he/he) ;

IV polydactyle $(+/$ he $) \times$ hémimélique (he/he) . I $(+/$ he $):$ I (he/he).
}

(1) Quatre séries de croisements seulement ont pu être effectuées, les máles he/he étant stériles. 
I. - ANALYSE GÉNÉTIQUE DES SOURIS HÉMIMÉLIQUES ET POLYDACTYLES: VÉRIFICATION DE LA DESCENDANCE NORMALE

I. Étude des résultats des quatre types de croisements fondamentaux (voir tabl. I).

Nous constatons à première vue, que la descendance dans le croisement de 1'expérience I (normal $\times$ hémimélique), au lieu d'être exclusivement composée de sujets polydactyles, comprend deux classes : polydactyles et normaux. Il semblerait que nous soyons en présence d'une nouvelle classe de descendants, non prévue par la théorie, composée de sujets d'apparence normale.

\section{TABLEAU I}

Résultats des qualres croisements fondamentaux dans les quatre milieux génétiques RAP, MO, C.3H et C57BL

\begin{tabular}{|c|c|c|c|c|c|}
\hline \multirow{2}{*}{$\begin{array}{l}\text { Parents } \\
\sigma \times 0\end{array}$} & \multirow{2}{*}{$\begin{array}{l}\text { Milieu } \\
\text { génétique }\end{array}$} & \multicolumn{3}{|c|}{ Descendants } & \multirow{2}{*}{$\begin{array}{c}\text { Total des } \\
\text { petits }\end{array}$} \\
\hline & & Normaux & Polydactyles & Hémiméliques & \\
\hline \multirow{4}{*}{$\begin{array}{c}\text { Expérience } I \\
\text { Normal } \times \text { Ifémimélique } \\
+/+\quad \text { he/he }\end{array}$} & RAI' & 97 & 56 & - & 153 \\
\hline & $\mathrm{MO}$ & 151 & 4 & - & 155 \\
\hline & $\mathrm{C} 3 \mathrm{II}$ & 52 & 10 & - & 62 \\
\hline & C57BL & 16 & 15 & - & 31 \\
\hline \multirow{4}{*}{$\begin{array}{c}\text { Expérience II } \\
\text { Polydactyle } \times \text { Normale } \\
+/ \text { he } \quad+/+ \\
\text { Normal } \times \text { Polydactyle } \\
+1+\quad+/ \text { he }\end{array}$} & RAP & 573 & 198 & - & 771 \\
\hline & $\mathrm{MO}$ & 952 & 93 & - & 10,5 \\
\hline & $\mathrm{C} 3 \mathrm{H}$ & 308 & 35 & - & 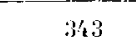 \\
\hline & $\mathrm{C} 57 \mathrm{BL}$ & 162 & 76 & - & 238 \\
\hline \multirow{4}{*}{$\begin{array}{c}\text { Expérience III } \\
\text { Polydactyle } \times \text { Polydactyle } \\
+/ \text { he } \\
+/ \text { he }\end{array}$} & RAP & 334 & 429 & 259 & 1022 \\
\hline & $\mathrm{MO}$ & 319 & 166 & 178 & 663 \\
\hline & $\mathrm{CoII}$ & 156 & $1 / 1$ & 90 & 387 \\
\hline & C57BI & $3 '$ & 35 & 21 & 93 \\
\hline \multirow{3}{*}{$\begin{array}{c}\text { Expérience IV } \\
\text { Polydactyle } \times \text { Hémimélique } \\
+/ \text { he he he }\end{array}$} & $\mathrm{RAP}$ & 37 & 60 & 72 & 169 \\
\hline & Mo & 18 & $3: 3$ & 49 & 100 \\
\hline & $\mathrm{C} 3 \mathrm{H}$ & 12 & 6 & 17 & 35 \\
\hline
\end{tabular}

Les croisements de l'expérience II (polydactyle $\times$ normal), conformément aux prévisions, fournissent deux classes de descendants : polydactyles et normaux; toutefois les descendants ne se répartissent pas statistiquement dans les proportions prévues ( $\mathrm{x}: \mathrm{I}$ ). Nous constatons un excédent de sujets d'apparence normale. 
Les croisements de l'expérience III (polydactyle $\times$ polydactyle), donnent bien trois classes de descendants : normaux, polydactyles, hémiméliques; cependant ils ne se répartissent pas statistiquement dans les porportions prévues (I: $2:$ I). Nous notons, ici aussi, un excédent de sujets d'apparence normale.

Dans les croisements de l'expérience IV (hémimélique $\times$ polydactyle), au lieu d'obtenir deux classes de descendants: polydactyles et hémiméliques dans les proportions $(\mathrm{I}: \mathrm{I}$ ), selon les prévisions, nous observons en outre la présence de sujets d'apparence normale, non prévus par la théorie.

En résumé, quel que soit le type de croisement effectué, nous obtenons des descendants d'apparence normale non prévus par la théorie (exp. I et IV) ou en excédent (exp. II et III). Dès lors quelle est leur constitution génotypique?

\section{Analyse de la constitution génotypique}

de la descendance d'apparence normale (voir tabl. 2)

Pour étudier le comportement génétique des descendants d'apparence normale issus de l'expérience $I$, trois types de croisements ont été effectués.

$\mathrm{V} \mathrm{F}_{1}$ Nov issu de l'exp. I $\times$ hémimélique.

VI $F_{1}$ Nov issu de l'exp. I $\times$ polydactyle.

VII $\mathrm{F}_{1}$ Nov issu de l'exp. I $\times \mathrm{F}_{1}$ Nov issu de l'exp. I.

Nous constatons que les descendants d'apparence normale $\left(\mathrm{F}_{1}\right.$ de l'expérience $\left.\mathrm{I}\right)$, se comportent, tous sans exception, comme des polydactyles; c'est-à-dire qu'ils sont hétérozygotes à phénotype non exprimé (normal overlaps). En effet dans l'expérience $\mathrm{V}$ nous avons obtenu trois classes de descendants, comme dans l'expérience IV. Mêmes résultats dans les expériences VI et VII.

En ce qui concerne les sujets normaux en excédent dans la descendance issue de l'expérience II, le problème est différent : alors que dans l'expérience I tout porte à croire que les descendants d'apparence normale étaient des hétérozygotes, dans l'expérience II la descendance devrait comprendre en dehors des sujets hétérozygotes d'apparence normale des sujets réellement normaux deconstitution génotypique $(+/+)$ : cinq types de croisements permettront de justifier cette hypothèse :

$\mathrm{VIII}_{a}$ polydactyle (+/he) $\times \mathrm{F}_{1}$ Nov $(+/$ he $)$ issue de l'exp. II.

$\mathrm{IX}_{a} \quad \mathrm{~F}_{1}$ Nov (+/he) du II $\times \mathrm{F}_{1}$ Nov $(+/$ he $)$ issue de l'exp. II.

$\mathrm{VIII}_{b}$ polydactyle $(+/ \mathrm{he}) \times \mathrm{F}_{1} N(+/+)$ issue de l'exp. II.

$\mathrm{IX}_{b} \quad \mathrm{~F}_{1}$ Nov du II $(+/$ he $) \times \mathrm{F}_{1} N(+/+)$ issue de l'exp. II.

$\mathrm{IX}_{c} \quad \mathrm{~F}_{1} N$ du II $(+/+) \times \mathrm{F}_{1} N$ du II $(+/+)$ issue de l'exp. II.

Nous constatons que les résultats des expériences $\operatorname{VIII}_{a}$ et $\operatorname{IX}_{a}$ sont semblables à ceux de l'expérience III, c'est-à-dire qu'ils donnent trois classes de descendants comme lors du croisement de deux polydactyles, ce qui montre bien que les géniteurs $\mathrm{F}_{1}$ Nov sont des hétérozygotes ( $+/ \mathrm{he}$ ).

I,es résultats des expériences $\mathrm{VIII}_{b}$ et $\mathrm{IX}_{b}$ sont les mêmes que ceux obtenus 
lors de l'expérience II, c'est-à-dire qu'ils donnent deux classes de descendants comme lors du croisement de sujets polydactyles avec des sujets normaux. Ici aussi, les géniteurs $\mathrm{F}_{1}$ Nov se sont comportés comme des hétérozygotes ( $+/$ he).

TABIEAU 2

Analyse de la constitution ginotypique de la descendance d'apparence normale issue des expériences I et II

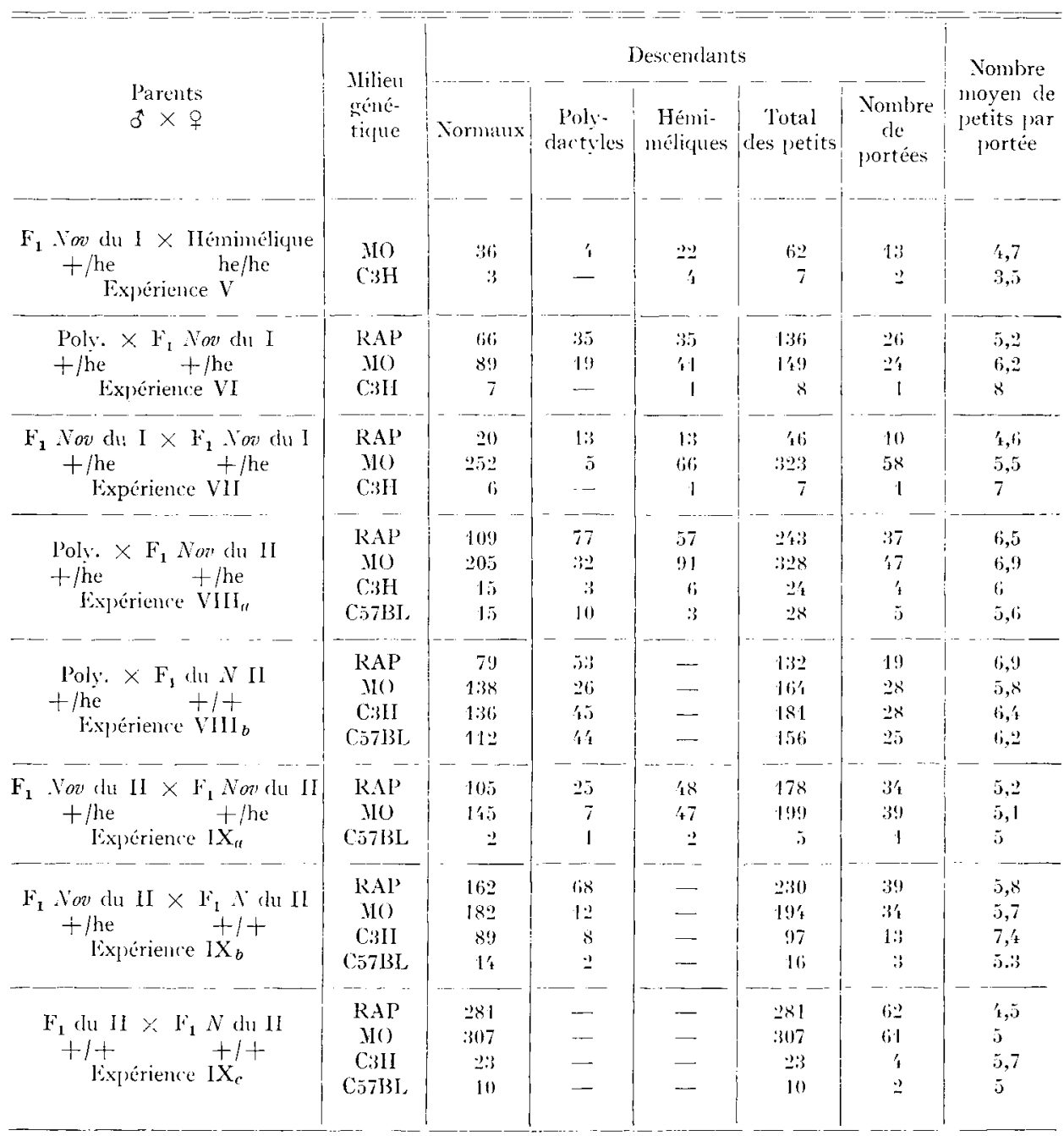

Abréviations: Poly. $=$ Polvdactyle; . Von $=$ Vormal overlaps; $\mathrm{N} .=$ normal.

Enfin, dans les croisements de l'expérience $I_{c}$, entre géniteurs supposés normaux, issus de l'expérience II, nous avons obtenu exclusivement des descendants normaux, ce qui montre qu'ils étaient de constitution génotypique $(t / t)$. 
Conclusion. - Les souris hémiméliques sont de constitution génotypique (he/he): homozygotes et les polydactyles de même que les sujets normal overlaps de constitution génotypique $(+/$ he $)$ : hétérozygotes.

\section{II. - ÉTUDE DE I.A PÉNÍTRANCE}

\section{A - Pénétrance chez les homozygotes hémiméliques}

Dans les croisements de sujets homozygotes hémiméliques avec des hétérozygotes polydactyles ou normal overlaps c'est-à-dire d'hétérozygotes à phénotype exprimé ou non (exp. IV et V), la répartition des homozygotes dans la descendance est toujours dans les proportions : (II) quand on groupe les polydactyles et les normal overlaps. (tabl 3).

TABLEAU 3

Pénétrance des homozygotes hémiméliques dans la descendance issue des croisements $($ he/he $) \times(+/$ he $)$ : expériences $I V$ et $V$

\begin{tabular}{|c|c|c|c|c|}
\hline \multirow{2}{*}{$\begin{array}{l}\text { Nilieu } \\
\text { génćtique }\end{array}$} & \multicolumn{3}{|c|}{ Répartition des clesteudants } & $\left(n_{i}\right)^{2}$ \\
\hline & $\begin{array}{c}\text { Normal overlaps }+ \\
\text { Polydactyles } \\
\left(x_{i}\right)\end{array}$ & $\begin{array}{l}\text { Jéminúliques } \\
\qquad\left(n_{i}-x_{i}\right)\end{array}$ & $\begin{array}{l}\text { Total } \\
\left(n_{i}\right)\end{array}$ & $\chi_{1}^{2}=\frac{1}{\frac{1}{2} \cdot \frac{1}{2} \cdot n_{i}}$ \\
\hline $\mathrm{RAP}$ & $37+60=97$ & 72 & 169 & $\chi_{1}^{2}=3, y^{\prime}$ non significatif \\
\hline MO & $51+37=91$ & 71 & $16:$ & $\chi_{1}^{2}=2,4$ non significatif \\
\hline C:3H & $15+6=21$ & 21 & 42 & $x_{1}^{2}=0$ non significatif \\
\hline
\end{tabular}

\section{TABLEAU 4}

Pénétrance des homozygotes hémiméliques dans la descendance issue des croisemenls $(+/$ he $) \times(+/$ he $):$ expiriences $I I I, V I, V I I, V I I I_{\mathrm{a}}$ et $I X_{\mathrm{a}}$

\begin{tabular}{|c|c|c|c|c|}
\hline \multirow{2}{*}{$\begin{array}{l}\text { Milieu } \\
\text { génétique }\end{array}$} & \multicolumn{3}{|c|}{ Répartition des descendants } & ;) \\
\hline & $\begin{array}{c}\text { Nomaux ou Nov }+ \\
\text { Polydactyles } \\
\left(x_{i}\right)\end{array}$ & $\begin{array}{l}\text { Ilémiméliques } \\
\qquad\left(n_{i}-x_{i}\right)\end{array}$ & $\begin{array}{l}\text { Total } \\
\left(n_{i}\right)\end{array}$ & $\chi_{1}^{2}=\frac{1}{\frac{1}{1} \cdot n_{i}^{\prime} \cdot n_{i}}$ \\
\hline RAP & $633^{\prime}+579=1213$ & $41: 2$ & 1625 & $\chi_{1}^{2}=0,11$ non significatif \\
\hline $\mathrm{MO}$ & $1010+239=1239$ & $\left\{\begin{array}{l}9 \\
2\end{array}\right.$ & $166 ; 2$ & $\chi_{1}^{2}=0,20$ non signiticatif \\
\hline $\mathrm{C} 3 \mathrm{H}$ & $184+14 t=328$ & וs & 126 & $\chi_{1}^{2}=0,89$ non significatif \\
\hline Co7BL & $51+46=97$ & 29 & 126 & $\chi_{1}^{2}=0,25$ non signiticatif \\
\hline
\end{tabular}


230 N. KOBOZIEFF, N. POMRIASKINSKY-KOBOZIEFF, E. GEMÄHLING, F. REYNĖS

- Dans le croisement hétérozygote $\times$ hétérozygote, quele phénotype soit exprimé ou non (exp. III, VI, VIII ${ }_{a}$, IX $_{a}$ ), l'ensemble (normaux + hétérozygotes) et les homozygotes sont toujours dans le rapport : (3I) (tabl. 4).

La pénétrance est donc absolue pour les homozygotes, quel que soit le milieu génétique.

\section{$\mathrm{B}$ - Itude de la pénétrance chez les hétérozygotes polydactyles}

Pour étudier la fréquence d'apparition de sujets polydactyles, nous avons effectué quatre séries de croisements :

I. Normal $(+1+) \times$ homozygote (he/he). Exp. I.

II. Normal $(+/+) \times$ hétérozygote $\left(+/\right.$ he). Exp. $\mathrm{II}_{a}, \mathrm{II}_{b}, \mathrm{VIII}_{b}$ et $\mathrm{IX}_{b}$.

III. Hétérozygote $(+/$ he $) \times$ hétérozygote $(+/$ he $)$. Exxp. III, VI, VII, VIII $a$ et IX .

IV. Hétérozygote $(+/$ he $) \times$ homozygote (he/he). Exp. IV et V.

I. Résultats des croisements de la série $I$ ( $\sigma^{x}$ normal $\times$ q hémimélique) (tabl. 5)

Nous constatons que la pénétrance de la polydactylie varie suivant le milieu génétique des mâles normaux introduit dans le patrimoine héréditaire des mutants (Prob. $\left[\chi_{3}^{2}>68,6\right]<0,001$ )

Elle est forte dans le milieu $\mathrm{C}_{57} \mathrm{BL}$ et RAP, sans qu'on puisse toutefois les distinguer (respectivement 48,4 p. Ioo et 36,6 p. Ioo : $\chi_{1}^{2}=1,47$ non significatif) ; plus faible dans le milieu $\mathrm{C}_{3} \mathrm{H}$ ( 19,4 p. IOo), et très faible dans le milieur $\mathrm{MO}(2,5$ p. IOO).

\section{TABLEAU 5}

Milieu génétique et pénétrance dans les croisements de la série 1

(ơ normal $\times$ o hémimélique : expérience I)

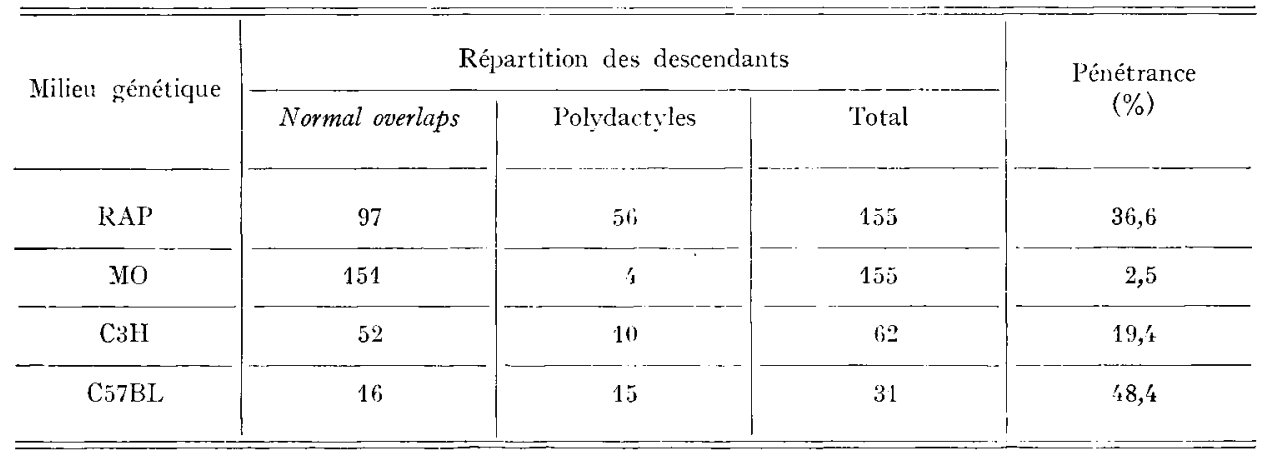

2. Résultats des croisements de la série II

(entre sujets normaux et hétérozygotes normal overlaps ou polydactyles)

Sans tenir compte du degré d'expressivité des sujets polydactyles.

Nous avons procédé à quatre expériences de croisements, nous permettant de rechercher s'il existait :

a) une influence du milieu génétique des souris normales introduit dans le patrimoine héréditaire des mutants; 
b) une influence du sexe de ces géniteurs normaux;

c) une différence de la pénétrance entre la première génération et celle issue de croisement de retour $\left(\mathrm{F}_{1}\right.$ et $\left.\mathrm{F}_{b}\right)$;

d) une influence des géniteurs hétérozygotes polydactyles et normal overlaps.

a) Infuence du milieu génétique des souris normales introduit dans le patrimoine héréditaire des mutants (voir tabl. 6).

D'une façon générale, dans cette série de croisements, le milieu génétique des souris normales introduit dans le patrimoine héréditaire des mutants, a une influence certaine sur la variation de la pénétrance (Prob. $\left[\chi_{3}^{2}>\mathrm{IgO}, 4\right] \ll 0,00 \mathrm{I}$ ).

\section{TABIEAU 6}

Milieu génétique et pénétrance dans les croisements de la série II (normal $\times$ hétérozygote : expériences $I I_{\mathrm{a}}, I I_{\mathrm{b}}, V I I I_{\mathrm{b}}$ et $I X_{\mathrm{b}}$ )

\begin{tabular}{|c|c|c|c|c|}
\hline \multirow{2}{*}{$\begin{array}{l}\text { Milieu } \\
\text { génétique }\end{array}$} & \multicolumn{3}{|c|}{ Répartition de la descendance } & \multirow{2}{*}{$\begin{array}{c}\text { Pénétrance } \\
(\%)\end{array}$} \\
\hline & $\begin{array}{l}\text { Normaux } \\
\text { ou Nov' }\end{array}$ & Polydactyles & Total & \\
\hline $\mathrm{RAl}^{\prime}$ & 814 & 219 & 1133 & 56,3 \\
\hline MO & 1272 & 131 & 1403 & 18,6 \\
\hline $\mathrm{C} ; 3 \mathrm{H}$ & 533 & 88 & 621 & 98,3 \\
\hline C57BI & 288 & 122 & 110 & 59,6 \\
\hline
\end{tabular}

Elle est forte pour les milieux $\mathrm{RAP}$ et $\mathrm{C}_{57} \mathrm{BL}$ avec respectivement 56,3 et 59,6 p. Ioo (sans que 1'on puisse toutefois les distinguer : $\chi_{1}^{2}=0,38$ ); plus faible dans le milieu $\mathrm{C}_{3} \mathrm{H}(28,3 \mathrm{p}$. Ioo) et très faible dans le milieu génétique $\mathrm{MO}$ (I8,6 p. Ioo)

b) Infuence du sexe des géniteurs normaux (voir tabl. 7).

En effectuant une première comparaison des résultats des expériences $\mathrm{II}_{\boldsymbol{a}}$ ( $\sigma^{x}$ polydactyle $\times \subsetneq$ normale) et $\mathrm{II}_{b}$ ( $\sigma^{x}$ normal $\times q$ polydactyle), sans prendre en considération le degré d'expressivité de l'anomalie chez ces derniers, nous observons que lorsque le géniteur normal appartient à la lignée RAP, la pénétrance est statistiquement la même que ce soit le mâle ou la femelle qui représente la lignée normale : 50,3 et 52,4 p. IOo respectivement : $\chi_{1}^{2}=0,09$ non significatif ; il en est de même pour la lignée $\mathrm{C}_{3} \mathrm{H}$ avec respectivement 23,3 et $\mathrm{I} 6,4$ p. Ioo : $\chi_{1}^{2}=\mathrm{I}, 03$ non significatif.

$\mathrm{Si}$, pour ces mêmes croisements, nous utilisons des géniteurs normaux de la lignée MO, la pénétrance est significativement plus faible quand c'est le mâle qui est normal I3, I et 22,5 p. IOO respectivement : $\left(\chi_{1}^{2}=7\right.$, I4 et $\mathrm{P}<0,0$ I). Par contre, dans les croisements où nous utilisons des géniteurs normaux de la lignée 
$\mathrm{C}_{57} \mathrm{BL}$, la pénétrance est plus faible quand c'est la femelle qui est normale : respectivement 43,8 et 77,4 p. IOO $\left(\chi_{1}^{2}=7,56\right.$ et $\left.\mathrm{P}<0,00 \mathrm{I}\right)$.

\section{TABLEAU 7}

Infuence du sexe des giniteurs normaux

\begin{tabular}{|c|c|c|c|c|c|}
\hline \multirow{2}{*}{$\begin{array}{c}\text { Milieu } \\
\text { génétique }\end{array}$} & \multirow[b]{2}{*}{ Parents $0 \times 9$} & \multicolumn{3}{|c|}{ Répartition des descendants } & \multirow{2}{*}{$\begin{array}{c}\text { l'énétrance } \\
(\%)\end{array}$} \\
\hline & & 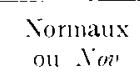 & $\begin{array}{l}\text { Poly- } \\
\text { dactiles }\end{array}$ & Total & \\
\hline \multirow{2}{*}{ RAP } & I'olydactyle $\times$ Normale. IXxp. II ${ }_{a} \mid$ & 300 & 101 & 101 & $.00,3$ \\
\hline & Normal $\times$ Polydactyl. Exp. $\mathrm{II}_{b}$ & $27: 3$ & 47 & 370 & 52,4 \\
\hline \multirow{2}{*}{$\mathrm{MO}$} & Polydactyle $\times$ Nomale. I:x] . II $a$ & i.sit & $5 x$ & $s L^{\prime}$ & $2-1,5$ \\
\hline & Cormal $\times$ Polydactyle. Exp. $\mathrm{II}_{b}$ & 496 & 35 & $5: 31$ & $1: 3,1$ \\
\hline \multirow{2}{*}{$\mathrm{C} 3 \mathrm{H}$} & Polydartyle $\times$ Normale. Exp. $\mathrm{II}_{a}$ & 17 & $\because 3$ & 197 & $\because 3,3$ \\
\hline & Normal $\times$ Polyclactyle. Exp. $I_{b}$ & $1: 34$ & 1른 & $1,6 i$ & $16, i$ \\
\hline \multirow{2}{*}{$\mathrm{C} . \mathrm{BL}$} & Polyclactyle $x$. Commale. Exp. Il & 7i) & $\because 1$ & 96 & i:s, \\
\hline & Normal $\times$ Polydartyle. Exp. II & $x \overline{7}$ & is & $14: 2$ & 77,1 \\
\hline
\end{tabular}

Retenons donc de cette première comparaison que les géniteurs normaux mâles et femelles ont une action similaive sur la pénétrance dans les milieux génétiques RAP et $\mathrm{C}_{3} \mathrm{H}$; par contre, dans le milieu MO le croisement $\sigma^{x}$ normal $\times q$ polydactyle donne une pénétrance plus faible que dans le croisement réciproque; enfin, dans le milieu $\mathrm{C}_{57} \mathrm{BL}$ c'est le croisement $q$ normale $\times \sigma^{x}$ polydactyle qui donne une pénétrance plus faible que le croisement réciproque.

c) Différence de la pénétrance entre la première génération et celle issue du croisement de retour $\left(F_{1}\right.$ et $\left.F_{l}\right)$ (voir tabl. 8).

Si l'on observe les résultats des croisements des expériences $\mathrm{IL}_{\text {" }}$ ( $\sigma^{\top}$ polydactyle $\times q$ normale $)$ et $\mathrm{VIII}_{b}\left(\sigma^{*}\right.$ polydactyle $\times \Varangle F_{1}$ normale issue de l'expérience $\left.I I\right)$, c'est-à-dire si l'on compare la pénétrance en $\mathrm{F}_{1}$ avec celle observée en $\mathrm{F}_{b}$, on constate que les résultats sont, une fois encore, différents suivant le milieu génétique étudié. Ainsi, pour le milieu génétique $\operatorname{RAP}$ la pénétrance est de $80,2 \mathrm{p}$. Ioo en $\mathrm{F}_{b}$ contre 50,3 p. Ioo en $F_{1}$, la différence est significative (Prob. $\left[\chi_{I}^{2}>10,9\right]<0,00 I$ ). Il en est de même pour le milieu génétique $\mathrm{C}_{3} \mathrm{H}$ où la pénétrance est de $49.7 \mathrm{p}$. Ioo en $\mathrm{F}_{b}$ contre 22,3 p. Ioo en $\mathrm{F}_{1}$ (Prob. $\left[\chi_{1}^{2}=\mathrm{II}, \mathrm{I}\right]<0, \mathrm{OOI}$ ).

$\mathrm{Au}$ contraire, pour les deux milieux génétiques $\mathrm{MO}$ et $\mathrm{C}_{57} \mathrm{BL}$, avec une pénétrance de $3 I, 7$ p. xoo en $F_{b}$ contre 22,5 p. Ioo en $F_{1}$ et 56,4 p. IOO en $F_{b}$ et 43,8 p. IOO en $F_{1}$ respectivement ; la différence n'est pas significative : $\chi_{1}^{2}=2,4$ et $: \chi_{1}^{2}=1,3$.

Constate-t-on encore une variation de la pénétrance, suivant le milieu génétique pour les descendances en $F_{1}$ et en $F_{b}$ étudiées séparément? 
En $\mathrm{F}_{1}$ la différence de la pénétrance entre les milieux RAP, MO, $\mathrm{C}_{3} \mathrm{H}$ et $\mathrm{C}_{57} \mathrm{BL}$, est significative (Prob. $\left[\chi_{1}^{2}>36,9\right]<0,00 \mathrm{I}$ ); il semble que l'on puisse distinguer d'une part, un ensemble à faible pénétrance : $\mathrm{MO}$ et $\mathrm{C}_{3} \mathrm{H}$ (de l'ordre de $22 \mathrm{p}$. I00), et d'autre part, un ensemble à forte pénétrance RAP et $\mathrm{C}_{57} \mathrm{BL}_{4}$ (de l'ordre de $45 \mathrm{p}$. I0o).

En $F_{b}$, s'il est vrai que l'on constate encore une variation de la pénétrance suivant le milieu génétique (Prob. $\left[\chi_{3}^{2}=22,6\right]<0,001$ ), la distribution des ensembles n'est pas la mème; on va d'un milieu à faible pénétrance: MO (3I,7 p. IOo) à un milieu à très forte pénétrance : $\operatorname{RAP}(80,2 \mathrm{p}$. IOo) en passant par un ensemble intermédiaire comportant deux milieux génétiques $\mathrm{C}_{3} \mathrm{H}$ et $\mathrm{C}_{57} \mathrm{BL}$ avec une pénétrance de l'ordre de 52 p. Ioo.

TABLEAU 8

Pénétrance en $F_{1}\left(E_{x p} . I I_{\mathrm{a}}\right)$ el $F_{\mathrm{b}}\left(\operatorname{Exp} . V I I I_{\mathrm{b}}\right)$

\begin{tabular}{|c|c|c|c|c|c|c|}
\hline \multirow[b]{2}{*}{$\begin{array}{l}\text { Milieu } \\
\text { grénétique }\end{array}$} & \multirow[b]{2}{*}{ Parents $\vec{\delta} \times q$} & \multirow[b]{2}{*}{$\begin{array}{l}\text { Généra- } \\
\text { tions }\end{array}$} & \multicolumn{3}{|c|}{ Répartition des descendants } & \multirow[b]{2}{*}{$\begin{array}{c}\text { Pénétrance } \\
(\%)\end{array}$} \\
\hline & & & $\begin{array}{l}\text { Normaux } \\
\text { ou nowmal } \\
\text { oyerlaps }\end{array}$ & $\begin{array}{c}\text { Poly- } \\
\text { dactyles }\end{array}$ & Total & \\
\hline \multirow{2}{*}{$\mathrm{RAP}$} & Polvdactyle $\times$ Normale & $\mathrm{F}_{1}$ & 300 & 101 & 101 & $50, .3$ \\
\hline & Poly $\times \mathrm{F}_{1}$ Normale issue du II & $\mathrm{T}_{b}$ & 79 & 53 & 132 & 80,2 \\
\hline \multirow{2}{*}{ 110 } & Polydactyle $\times$ Normale & $\mathrm{F}_{1}$ & 456 & 58 & $511^{\prime}$ & 22,5 \\
\hline & Poly. $\times F_{1}$ Normale issue du II & $\mathrm{I}_{b}$ & $13 x^{2}$ & 26 & $166^{\prime}$ & $: 31,7$ \\
\hline \multirow{2}{*}{ CsII } & Polydactyle $\times$ Normale & $\mathrm{F}_{1}$ & 174 & 23 & 197 & 23,3 \\
\hline & Poly. $\times F_{1}$ Normalc issue du II & $\mathrm{F}_{b}$ & 136 & 45 & 181 & $(9), 7$ \\
\hline \multirow{2}{*}{$\mathrm{C} 57 \mathrm{BL}$} & Polydactyle $\times$ Normale & $\mathrm{F}_{1}$ & 75 & 21 & 96 & 13,8 \\
\hline & Poly. $\times F_{1}$ Normale issue du II & $\mathrm{F}_{b}$ & 112 & $44^{\prime}$ & 156 & 56,4 \\
\hline
\end{tabular}

Examinons ces résultats de plus près, en comparant les distributions des pénétrances en $\mathrm{F}_{1}$ et $\mathrm{F}_{b}$; elles se présentent schématiquement de la façon suivante :

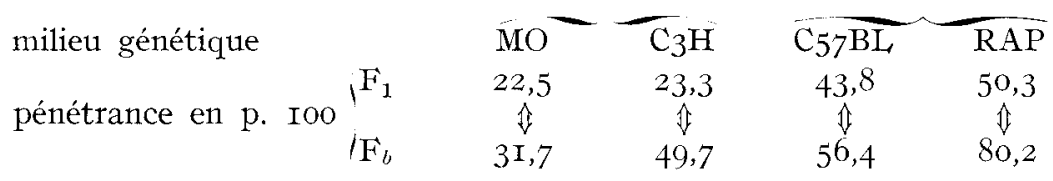

Si la pénétrance seule entrait en jeu, on devrait retrouver en $\mathrm{F}_{b}$ la même distribution des taux de pénétrance qu'en $\mathbf{F}_{1}$ or, ce n'est pas le cas. On a essayé d'expliquer cette différence, favorisant l'apparition de l'anomalie, par l'intervention de gènes modificateurs, dont l'accumulation serait rapide pour les milieux génétique RAP et $\mathrm{C}_{3} \mathrm{H}$ et non décelable au niveau des milieux $\mathrm{C}_{57} \mathrm{BL}$ et $\mathrm{MO}$; on remarquera que ce phénomène se produit, que la pénétrance soit forte $(\mathrm{RAP})$ ou faible $\left(\mathrm{C}_{3} \mathrm{H}\right)$. Il est fort possible, qu'en renouvelant les croisements de retour, on puisse mettre en évidence le même phénomène pour les milieux $\mathrm{C}_{5} 7 \mathrm{BL}$ ou MO 
234 N. KOBOZIEFF, N. POMRIASKINSKY-KOBOZIEFF, E. GEMÄHLING, F. REYNÈS

d) Influence des géniteurs hétérozygotes polydactyles et normal overlaps (voir tabl. 9).

Nous avons aussi voulu savoir s'il existait une différence dans la pénétrance chez les descendants issus du croisement d'hétérozygotes chez lesquels le phénotype est exprimé : sujets polydactyles et ceux issus du croisement d'hétérozygotes chez lesquels le phénotype n'est pas exprimé : sujets normal overlaps. Dans ce but nous avons comparé les résultats des expériences de croisement $\operatorname{VIII}_{b}$ ( $\sigma^{x}$ polydactyle $\times \subsetneq F_{1}$ normale issue $\left.d u I I\right)$ et $\operatorname{IX}_{b}\left(F_{1}\right.$ normal overlaps $i s s u d u I I \times F_{1}$ normale issue du II).

TABLEAU 9

Infuence des géniteurs hétérozygotes polydactyles el normal overlaps sur la pénétrance

\begin{tabular}{|c|c|c|c|c|c|}
\hline \multirow{2}{*}{$\begin{array}{l}\text { Parents } \\
\jmath \times \emptyset\end{array}$} & \multirow{2}{*}{$\begin{array}{c}\text { Milieu } \\
\text { Génétique }\end{array}$} & \multicolumn{3}{|c|}{ Répartition de la descendance } & \multirow{2}{*}{$\begin{array}{l}\text { Pénétrance de } \\
\text { la polydactylie } \\
(\%)\end{array}$} \\
\hline & & $\left|\begin{array}{c}\text { Normaux } \\
\text { ou } N o v\end{array}\right|$ & $\begin{array}{c}\text { Poly- } \\
\text { dactyles }\end{array}$ & Total & \\
\hline Poly. $\times F_{1}$ Normale du II, exp. VIII $_{b}$ & \multirow{2}{*}{ RAP } & 79 & 53 & 132 & 80,2 \\
\hline $\begin{array}{l}\mathrm{F}_{1} \text { Nov du II } \times \mathrm{F}_{1} \text { Normale du II. } \\
\mathrm{F}_{1} \text { Normal du II } \times \mathrm{F}_{1} \text { Nov du II, exp. IX }\end{array}$ & & 162 & 68 & 230 & 59,1 \\
\hline Poly. $\times \mathbf{F}_{1}$ Normale du II exp. VIII $b$ & \multirow{2}{*}{$\mathrm{MO}$} & 138 & 26 & $16^{\prime}:$ & 31,7 \\
\hline $\begin{array}{c}\mathrm{F}_{\mathrm{I}} \text { Nov du II } \times \mathrm{F}_{1} \text { Normale du II } \\
\mathrm{F}_{1} \text { Normal du II } \times \mathrm{F}_{1} \text { Nov du II, exp. IX }\end{array}$ & & 182 & 12 & $19^{\prime} t$ & 12,3 \\
\hline Poly. $\times F_{1}$ Normale du II, exp. VIII $b$ & \multirow{2}{*}{$\mathrm{C} 3 \mathrm{H}$} & 136 & 45 & 181 & 49,7 \\
\hline $\begin{array}{c}\mathrm{F}_{\mathrm{I}} N o v \text { du II } \times \mathrm{F}_{1} \text { Normale du II } \\
\mathrm{F}_{1} \text { Normal du II } \times \mathrm{F}_{1} \text { Nov du II, exp. IX }\end{array}$ & & 89 & 8 & 97 & 16,4 \\
\hline Poly. $\times F_{1}$ Normale du II, exp. VIII $b$ & \multirow{2}{*}{ C57BL } & 112 & 44 & 156 & 56,4 \\
\hline $\begin{array}{l}\mathrm{F}_{1} \text { Nov du } \mathrm{II} \times \mathrm{F}_{1} \text { Normale du II } \\
\mathrm{F}_{1} \text { Nornal du } \mathrm{II} \times \mathrm{F}_{1} \text { Nov du II, exp. IX }\end{array}$ & & $1 / 4$ & 2 & 16 & 25 \\
\hline
\end{tabular}

Quel que soit le milieu génétique, la pénétrance est statistiquement plus faible quand l'un des géniteurs est un hétérozygote normal overlaps. Elle est de 59, I p. Ioo contre 80,2 p. IOO dans le milieu génétique $\operatorname{RAP}$ (Prob. $\left[\chi_{1}^{2}>6,25\right]<0,05$ ) pour $\mathrm{C}_{3} \mathrm{H}$ elle est de $16,4 \mathrm{p}$. IOo contre 49,7 p. IOo (Prob. $\left[\chi_{1}^{2}>8,77\right]<0,0 \mathrm{I}$ ), pour MO de I2,3 p. IOo contre 3I,7 p. IOo (Prob. $\left[\chi_{1}^{2}>\right.$ II,3I $]<0,00 I$ ) et pour $\mathrm{C}_{57}$ BL, elle est de $25 \mathrm{p}$. Ioo contre $56,4 \mathrm{p}$. IOo (Prob. $\left[\chi_{1}^{2}>4,4\right]<0,05$ ), on pourrait détecter aussi l'influence du milieu génétique que ce soit pour l'expérience $\mathrm{VIII}_{b}$ ou $\mathrm{IX}_{b}$ : respectivement (Prob. $\left[\chi_{6}^{2}>22,7\right]<0,00 I$ ) et (Prob. $\left[\chi_{3}^{2}>48,2\right]<0,00 I$ ).

En tenant compte du degré d'expressivité de l'anomalie chez les géniteurs polydactyles dans les croisements des expériences $I I_{a}$ et $I I_{b}$ (voir tabl. Io)

Notre analyse serait pourtant incomplète si nous ne tenions pas compte du degré d'expressivité de l'anomalie chez les géniteurs polydactyles (polydactylie bilatérale : expressivité forte, ou polydactylie unilatérale gauche ou droite : expres- 
sivité faible). Pour cette étude, nous avons pris en considération les deux milieux génétiques les mieux représentés : RAP et MO.

Pour le milieu RAP, dans le croisement de l'expérience $\mathrm{II}_{b}$ entre $\sigma^{x}$ normal $\times$ + polydactyle à faible ou forte expressivité, la pénétrance est statistiquement plus faible quand les femelles sont atteintes de polydactylie unilatérale gauche ou droite:

\section{TABLEAU IO}

Influence du degré d'expressivilé de l'anomalie chez les géniteurs polydactyles sur la pénétrance

\begin{tabular}{|c|c|c|c|c|c|}
\hline \multirow{2}{*}{$\begin{array}{l}\text { Milieu } \\
\text { génétique }\end{array}$} & \multirow{2}{*}{$\begin{array}{l}\text { Parents } \\
\delta \times 9\end{array}$} & \multicolumn{3}{|c|}{ Répartition des descendants } & \multirow[b]{2}{*}{$\begin{array}{l}\text { Pénétrance } \\
(\%)\end{array}$} \\
\hline & & $\begin{array}{l}\text { Normaux ou } \\
\text { normal overlaps }\end{array}$ & Polydactyles & Total & \\
\hline \multirow{5}{*}{ RAP } & Poly. bil. $\times$ Normale. Exp. $\mathrm{II}_{a}$ & 190 & 63 & 253 & 51,8 \\
\hline & $\begin{array}{l}\text { Poly. g. } \times \text { Normale. Exp. } \boldsymbol{I}_{a} \\
\text { Poly. d. }\end{array}$ & 120 & 38 & 158 & 48,1 \\
\hline & Normal $\times$ Poly. bil. Exp. $I_{b}$ & 180 & $8 \mathbf{t}^{\prime}$ & 264 & 63,6 \\
\hline & Normal $\times \frac{\text { Poly. g. Exp. } \mathrm{II}_{b}}{\text { Poly. d. }}$ & 93 & 13 & 106 & $24, \overline{5}$ \\
\hline & Total & 573 & 198 & 771 & 51,3 \\
\hline \multirow{5}{*}{$\mathrm{MO}$} & Poly. bil. $\times$ Normale. Exp. $\mathrm{II}_{a}$ & 268 & 35 & 305 & 24,2 \\
\hline & $\begin{array}{l}\text { Poly. g. } \times \text { Normale. Exp. } \mathrm{II}_{\alpha} \\
\text { Poly. d. }\end{array}$ & 188 & 21 & 209 & 20,0 \\
\hline & Normal $\times$ Poly. bil. Exp. $\mathrm{II}_{b}$ & 332 & 27 & 359 & 15,0 \\
\hline & Normal $\times \begin{array}{l}\text { Poly. g. } \\
\text { Poly. d. }\end{array}$ & 164 & 8 & 172 & 9,3 \\
\hline & 'Total & 952 & 93 & 1045 & 17,7 \\
\hline
\end{tabular}

$$
\text { Abréviations utilisées }\left\{\begin{array}{l}
\text { Poly. bil. = polydactylie bilatérale : expressivité forte } \\
\text { Poly. g. }=\text { polydactylie unilaté- } \\
\text { rale gauche } \\
\text { Poly. d. }=\begin{array}{l}
\text { polydactylie unilaté- } \\
\text { rale droite }
\end{array}
\end{array}\right\} \text { expressivité faible }
$$

expressivité faible $(24,5$ p. IOo) que quand elles sont atteintes plus gravement (polydactylie bilatérale : expressivité forte $63,6 \mathrm{p}$. I00) $\left(\chi_{1}^{2}=\mathrm{I} 5 ; \mathrm{P}<0,00 \mathrm{I}\right)$. Au contraire, lors $\mathrm{du}$ croisement $\bigcirc$ normale $\times \sigma^{x}$ polydactyle (expérience $\mathrm{II}_{a}$ ), l'expressivité de l'anomalie chez les mâles n'a aucune action sur la pénétrance 5I,8 p. Ioo quand 
le géniteur mâle est atteint de polydactylie, contre 48 , I p. Ioo pour les mâles atteints de polydactylie unilatérale gauche ou droite $\left(\chi_{1}^{2}=0,04\right)$.

Dans les croisements $\sigma^{x}$ normal $\times q$ polydactyle bilatérale et $\sigma^{x}$ polydactyle bilatéral $\times q$ normale (exp. $\mathrm{II}_{\text {l/ }}$ et $\mathrm{II}_{11}$ ), les pénétrances ne sont pas statistiquement différentes : 63,6 p. IOO contre $5 \mathrm{I}, 8$ p. I00 $\left(\chi_{1}^{2}=2,9\right)$.

Par contre, pour ces mêmes expériences, dans le croisement $\sigma^{x}$ normal $\times \emptyset$ polydactyle gauche ou droite la pénétrance est statistiquement plus faible que dans le croisement $\sigma^{x}$ polydactyle gauche ou droit $\times \Varangle$ normale : respectivement $24,5 \mathrm{p}$. 100 et 48 , I p. Ioo (Prob. $\left[\chi_{1}^{2}>4,9 j<0,05\right.$ ).

\section{En est-il de même pour le milien MO?}

Dans le croisement $\sigma^{*}$ normal $\times$ Q polydactyle à faible expressivité nous observons une pénétrance de $9,3 \mathrm{p}$. Ioo contre I $_{5} \mathrm{p}$. Ioo dans le croisement $\sigma^{x}$ normal $\times q$ polydactyle à forte expressizité ; la différence n'est pas significative $\left(\chi_{1}^{2}=0,6\right)$.

Autrement dit, dans ce milieu génétique, le mâle normal joue le même rôle, qu'il soit croisé avec des femelles à faible ou à forte expressivité. Ce résultat se retrouve quand on prend des femelles normales (respectivement 20,0 p. Ioo et 24,2 p. IOo: $\left.\chi_{1}^{2}=\mathrm{I}, 5\right)$.

Enfin, on ne décèle pas de différence significative $\left(\chi_{1}^{2}=0,24\right)$ entre les pénétrances relatives aux croisements $\sigma^{x}$ polydactyle bilatéral $\times$ normale $(24,2$ p. IOo) et $\sigma_{\text {normal }} \times \&$ polydactyle bilatérale ( $15 \mathrm{p}$. Ioo). Par contre la différence entre les pénétrances est significative $\left(\chi_{1}^{2}=9,6 ; \mathrm{P}<0, \mathrm{OOI}\right)$ lors des croisements $\sigma^{x}$ polydactyle gauche ou droite $\times \mp$ normale $\left(20,0\right.$ p. Ioo) et $\sigma^{x}$ normal $\times \uparrow$ polydactyle gauche ou droite (9,3 p. Ioo).

Comparons maintenant ces résultats pour le milieu RAP avec ceux du tableau 7 , oì il n'était pas tenu compte du degré d'expressivité de l'anomalie chez les géniteurs polydactyles. On observait que la pénétrance était statistiquement la même que ce soit le mâle ou la femelle qui représente la lignée normale.

Au contraire ici où l'on tient compte du degré d'expressivité de l'anomalie chez la femelle polydactyle, on constate que le mâle normal inhibe l'apparition de l'anomalie si la femelle est atteinte de polydactylie à faible expressivité (unilatérale gauche ou droite).

Qu'en est-il dans le milieu MO où l'on observait déjà une pénétrance plus faible quand c'était le mâle qui était normal ? Ce résultat reste vrai, mais l'on peut préciser que ceci n'est décelable que lorsque la femelle est atteinte de polydactylie à faible expressivité.

En examinant ces différents croisements on constate que la pénétrance est statistiquement plus faible dans le milieu MO que dans le milieu RAP.

$$
\begin{aligned}
& \sigma^{*} \text { polydactyle bilatéral } \times q \text { normate } \\
& \chi_{1}^{2}=\mathrm{I}_{5}, 4 ; \text { Prob. }<0, \text { OOI } \\
& \sigma^{*} \text { normal } \times q \text { polydactyle bilatérale } \\
& \sigma \text { polydactyle gauche ou droit } x+9 \text { normale } \\
& \sigma^{x} \text { normal } \times q \text { polydactyle gauche ou droite } \\
& \begin{array}{l}
\chi_{1}^{2}=6 \mathrm{I}, 4 ; \text { Prob. } \ll 0,00 \mathrm{I} \\
\chi_{1}^{2}=\mathrm{I} 3, \mathrm{I} ; \text { Prob. }<0, \mathrm{OOI} \\
x_{1}^{2}=5,5 ; \text { Prob. }<0,05
\end{array}
\end{aligned}
$$

Ceci se confirme si l'on considère que l'ensemble de ces croisements n'en forme qu'un seul : la pénétrance est plus faible dans le milieu génétique $\mathrm{MO}$ avec $\mathrm{I} 7,7 \mathrm{p}$. IOO que dans le milieu RAP avec 5I,3 p. IOO (Prob. $\left[\chi_{1}^{2}=92,9\right] \ll 0,00 \mathrm{I}$ ). (fig. 2 ). 
Résultats des croisements de la série III (hétérozygotes entre eux)

(voir tabl. II et fig. 3).

a) Influence de l'expression du phénotype chez les géniteurs.

Que ce soit pour le milieu génétique RAP ou pour le milieu génétique MO, la pénétrance est liée au type de croisement effectué, respectivement $\left(\chi_{4}^{2}=69,6\right.$; Prob. $\ll$ o,OOI et $\chi_{4}^{2}=\mathrm{I} 56$; Prob. $\ll$ o,OOI).

Pour le milieu génétique RAP, lors du croisement de deux géniteurs polydactyles (expérience III), sans tenir compte du degré d'expressivité de l'anomalie chez ces derniers, la pénétrance est de 83,9 p. Ioo ; elle est de 57,5 p. Ioo quand l'un des géniteurs est polydactyle et l'autre normal overlaps (expériences VI et VIIIa), enfin elle n'est que de 33,9 p. Ioo quand les deux géniteurs sont des sujets normal overlaps (expériences VII et $\mathrm{IX}_{r}$ ).

TABI,EAU II

Expression du phénotype chez les géniteurs et pénétrance dans les croisements de la série III

\begin{tabular}{|c|c|c|c|c|c|c|}
\hline \multirow{2}{*}{$\begin{array}{l}\text { Milieu } \\
\text { géné- } \\
\text { tique }\end{array}$} & \multirow{2}{*}{$\begin{array}{l}\text { Parents } \\
3 \times q\end{array}$} & \multicolumn{4}{|c|}{ Répartition des descenclants } & \multirow{2}{*}{$\begin{array}{l}\text { Péné- } \\
\text { trance } \\
(\%)\end{array}$} \\
\hline & & $\begin{array}{l}\text { Normaux } \\
\text { ou } \text { Va }\end{array}$ & $\begin{array}{l}\text { Poly- } \\
\text { dactyles }\end{array}$ & $\begin{array}{l}\text { Hémi- } \\
\text { méliques }\end{array}$ & Total & \\
\hline \multirow{4}{*}{ RAP } & Poly $\times$ Poly, ex]. III & 3331 & 但?!? & 259 & 102.2 & 83,9 \\
\hline & $\begin{array}{l}\text { Poly. } \times F_{1} \text {. Vow issue du I, exp. VI } \\
\text { Poly. } \times \mathrm{F}_{1} \text { Non issue du II, exp. VIII }\end{array}$ & 17. & 112 & $9: 2$ & 379 & $57, \tilde{3}$ \\
\hline & $\begin{array}{l}F_{1} N o v \text { du I } \times F_{1} \text { Vov du } \mathrm{l} \text {, exp. VII } \\
\mathrm{F}_{1} N\left(v v \text { du! II } \times \mathrm{F}_{1} \text { Nov du II, exp. IX } a\right.\end{array}$ & 195 & 38 & iil & 292 & $: 33,9$ \\
\hline & Total & $6 i 3 i^{\prime}$ & 579 & $41: 2$ & 1625 & 71,2 \\
\hline \multirow{4}{*}{.10 } & Poly. $\times$ Poly., exp. III & 319 & $166 i$ & 178 & 463 & 50,0 \\
\hline & $\begin{array}{l}\text { Poly. } \times \mathrm{F}_{1} N o v \text { issue du } \mathrm{I} \text {, exp. VI } \\
\text { Poly. } \times \mathrm{F}_{1}-\mathrm{V} \text { ov issue du } 1 \mathrm{I} \text {, exp. VIII }{ }_{a}\end{array}$ & 294 & 51 & $13: 2$ & 477 & $\geqslant 1,3$ \\
\hline & $\begin{array}{l}\mathrm{F}_{1} \text { Nov du I } \times \mathrm{F}_{1} \text { Nov du I, exp. VII } \\
\mathrm{F}_{1} \text { Nov du II } \times \mathrm{F}_{1} \text { Nov du II, exp. IX } \text { IX }_{u}\end{array}$ & 397 & 12 & 11.3 & $52 \div$ & 4,6 \\
\hline & Total & 1010 & $2: 29$ & 423 & 1662 & 27.5 \\
\hline
\end{tabular}

Pour le milieu MO lors du croisement de l'expérience II, dans les mêmes conditions, la pénétrance est de $5^{\circ} \mathrm{p}$. Ioo quand les deux géniteurs sont polydactyles; elle est de $2 \mathrm{I}, 3 \mathrm{p}$. Ioo quand l'un des géniteurs est polydactyle et l'autre normal overlaps, enfin elle n'est que de 4,6 p. Ioo quand les deux géniteurs sont normal overlaps. 
238 N. KOBOZIEFF, N. POMRIASKINSKY-KOBOZIEFF, E. GEMHÄLING, F. REYNÈS

Soulignons ici, que la pénétrance est étroitement liée à l'expression phénotypique des géniteurs. Elle est forte pour la descendance de deux géniteurs à phénotype exprimé, plus faible quand le phénotype n'est exprimé que chez l'un d'entre eux et encore plus faible lorsque les deux géniteurs sont à phénotype non exprimé (normal overlaps).

\section{b) Influence du milieu génétique.}

Remarquons qu'ici encore, l'influence du milieu génétique se fait sentir : pénétrance faible pour le milieu MO et forte pour le milieu RAP :

Exp. III : $\sigma^{x}$ polydactyle $\times q$ polydactyle, deux géniteurs à phénotype exprimé, pénétrance: 83,9 p. Ioo et 50 p. roo (Prob. $\left[\chi_{2}^{2}>57,7\right]<0,00 \mathrm{I}$ ) hautement significatif.

Exp. VI et $\operatorname{VIII}_{\alpha}: \sigma^{x}$ polydactyle $\times q \mathrm{~F}_{\mathrm{I}}$ normal overlaps issue $\mathrm{du} \mathrm{I}$ ou du II, l'un des géniteurs est hétérozygote à phénotype exprimé, l'autre non, pénétrance : 57,5 p. roo et $2 \mathrm{I}, 3$ p. Ioo $\left(\chi_{-2}^{2}=49,4 ;\right.$ Prob. $\left.<0,00 \mathrm{I}\right)$.

Exp. VII et $\mathrm{IX}_{a}: \mathrm{F}_{1}$ normal overlaps issu du I ou du II $\times \subsetneq \mathrm{F}_{1}$ normal overlaps issue du I ou du II, les deux géniteurs sont des hétérozygotes à phénotype non exprimé, pénétrance : 33,9 p. Ioo et 4,6 p. Ioo $\left(\chi_{2}^{2}=6 \mathrm{I}, 6\right.$; Prob. $\left.<0,00 \mathrm{I}\right)$.

Ceci se confirme si l'on considère que l'ensemble de ces croisements n'en forme qu'un seul, pour le milieu génétique $\operatorname{RAP}$ la pénétrance est de $7 \mathrm{I}, 2 \mathrm{p}$. Ioo contre 27,5 p. Ioo pour le milieu génétique $\mathrm{MO}\left(\chi_{2}^{2}=237,3 ;\right.$ Prob. $\left.\ll 0,00 \mathrm{I}\right)$.

c) Influence du mâle normal overlaps.

Dans les expériences de croisements entre géniteurs atteints de polydactylie bilatérale et sujets normal overiaps, il nous a paru intéressant de rechercher si le fait que ce soit le mâle ou la femelle qui était normal overlaps avait une influence sur la pénétrance. Nous avons pu faire cette étude seulement sur la lignée RAP, les chiffres que nous possédons pour les autres milieux génétiques étant trop faibles pour permettre de tirer des conclusions valables.

Infuence du sexe du géniteur normal suerlapss sur la pénétrance, milieu RAP.

\begin{tabular}{|c|c|c|c|c|c|}
\hline \multirow{2}{*}{$\begin{array}{l}\text { Parents } \\
0 \times 9\end{array}$} & \multicolumn{4}{|c|}{ Réjartition des descendants } & \multirow{2}{*}{$\begin{array}{l}\text { Pénétrance } \\
(\%)\end{array}$} \\
\hline & Normaux & Poly. & Hémimél. & Total & \\
\hline $\begin{array}{l}\text { Poly. bil. } \times \mathrm{F}_{1} \text { Nov issue du I exp. VI } \\
\text { Poly. bil. } \times \mathrm{F}_{1} \text { Nov issue du II exp. VIII }{ }_{a}\end{array}$ & 81 & 78 & 48 & 207 & 75,3 \\
\hline $\begin{array}{l}\mathrm{F}_{1} \text { Nov issu du } \mathrm{I} \times \text { Poly. bil. exp. VI. } \\
\mathrm{F}_{1} \text { Nov issu du II } \times \text { Poly. bil. exp. VIII }\end{array}$ & \} & 16 & 18 & 81 & 39,5 \\
\hline
\end{tabular}

Nous avons donc étudié les résultats de croisements entre géniteurs hétérozygotes à phénotype non exprimé avec des géniteurs polydactyles à forte expressivité (exp. VI et VIII ${ }_{a}$ ). Nous constatons que les mâles "normal overlaps » inhibent l'apparition de la polydactylie. La pénétrance est beaucoup plus faible si c'est le mâle qui 
est normal overlaps et non la femelle : 39,5 p. Ioo contre 75,3 p. I0o, dans le croisement inverse. La différence est significative $\left(\chi_{2}^{2}=6,4 ;\right.$ Prob. $\left.<0,05\right)$.

d) Rôle du degré d'expressivité de l'anomalie chez les géniteurs polydactyles dans les expériences III (tabl. I2).

Cette première étude ne tient pas compte du degré d'expressivité de la polydactylie chez les géniteurs; or, certains géniteurs sont atteints de polydactylie bilatérale (expressivité forte), d'autres de polydactylie unilatérale gauche ou droite (expressivité faible). Nous avons pris en considération les milieux génétiques RAP et MO, les mieux représentés dans ces types de croisements.

TABLEAU I 2

Rôle du degré d'expressivité chez les géniteurs hétérozygotes polydactyles

des milieux génétiques RAP et MO dans les croisements de l'exp. III sur la pénétrance

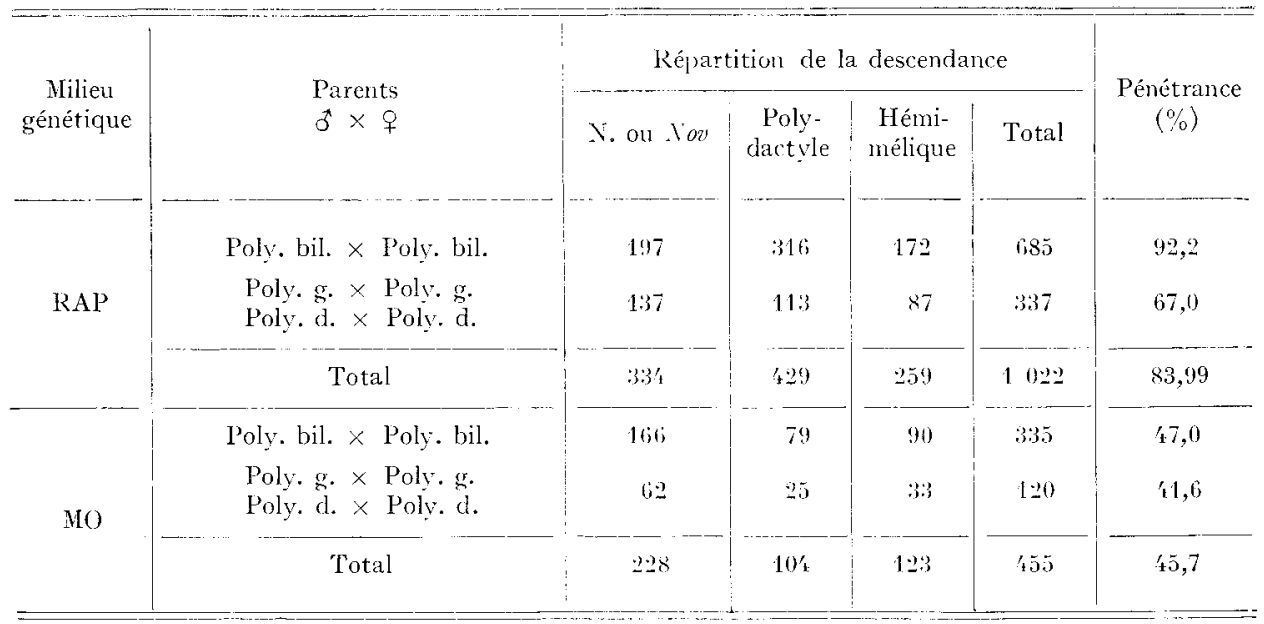

La pénétrance, dans la descendance issue du croisement de deux géniteurs à fort degré d'expressivité, est significativement plus forte $(92,2$ p. Ioo) que dans celle issue de deux géniteurs à faible degré d'expressivité $\left(67,0\right.$ p. Ioo) ; (Prob. $\left[\chi_{2}^{2}>\right.$ $\mathbf{I} 8,4]<0,00 \mathrm{I})$. Ceci, dans le milieu RAP. Dans le milieu génétique MO on ne distingue pas de différence entre les pénétrances respectives : 47,2 p. IoO contre 4I,6 p. IOO $\left(\chi_{2}^{2}=0,37\right)$.

Ici, encore, quel que soit le type de croisement (polydactylie bilatérale $\times$ polydactylie bilatérale ou polydactylie unilatérale $\times$ polydactylie unilatérale), la pénétrance est statistiquement plus forte dans le milieu génétique RAP que dans le milieu génétique MO.

poly. bil. $\times$ poly. bil.

$\left\{\begin{array}{l}\text { RAP 92,2 p. IOO. } \\ \text { MO 47,0 p. IOO }\end{array}\right.$

poly. unilat.
g. ou d. $\quad \begin{gathered}\text { poly. unilat. } \\ \text { g. ou d. }\end{gathered} \quad\left\{\begin{array}{l}\operatorname{RAP} 67,0 \text { p. IOO } \\ \operatorname{MO} 4 \mathrm{I}, 6 \text { p.0o. I }\end{array}\left(\chi_{2}^{2}=7,2 ;\right.\right.$ Prob. $\left.<0,05\right)$ 
240 N. KOBOZIEFF, N. POMRIASKINSKY-KOBOZIEFF, E. GEMAHLING, F. REYNìS

Ceci se confirme, quand on considère l'ensemble des résultats ; pour le milieu génétique RAP la pénétrance est de $83,9 \mathrm{p}$. IoO, elle n'est plus que de $45,7 \mathrm{p}$. Ioo dans le milieu MO $\left(\chi_{\mathrm{g}}^{2}=57,4\right.$; Prob. $<0$,OOI $)$.

Résultats des croisements de la série IV : o hétérozygote polydactyle $\times q$ homozygote hémimélique.

\begin{tabular}{|c|c|c|c|c|c|}
\hline \multirow{2}{*}{ Milieu génétique } & \multicolumn{4}{|c|}{ Répartition de la descendance } & \multirow{2}{*}{$\begin{array}{l}\text { Pénétrance } \\
\qquad(\%)\end{array}$} \\
\hline & Non & Polydactyle & Hémimélique & Total & \\
\hline $\operatorname{RAP} \ldots \ldots \ldots \ldots \ldots \ldots$ & 37 & (6) & 72 & 169 & 71,0 \\
\hline MO $\ldots \ldots \ldots \ldots \ldots \ldots$ & 18 & $3: 3$ & 69 & 100 & 66,0 \\
\hline
\end{tabular}

Nous constatons, que la pénétrance n'est pas absolue aussi bien dans le milieu génétique $\mathrm{RAP}$ que dans le milieu MO (7I,o p. Ioo et 66,o p. Ioo) ; on n'observe pas de différence statistique entre ces deux milieux : $\chi_{2}^{2}=r, 2$.

\begin{tabular}{|c|c|c|c|c|}
\hline & $Y a v$ & Polydactyle & Hémimélique & Total \\
\hline $\begin{array}{l}\text { o poly. } \times \text { q hémimélique } \ldots \ldots . \\
\delta \mathrm{F}_{1} \text { Nov issu du } \mathrm{I} \times \text { o hếni- } \\
\text { mélique } \ldots \ldots \ldots \ldots \ldots \ldots \ldots \ldots\end{array}$ & $\begin{array}{l}18 \\
36\end{array}$ & $\begin{array}{l}39 \\
\text { it }\end{array}$ & $\begin{array}{l}49 \\
29\end{array}$ & $\begin{array}{r}100 \\
6 \%\end{array}$ \\
\hline
\end{tabular}

Dans le croisement de l'expérience $V \sigma^{x}$ normal overlaps $\times q$ hémimélique on observe, pour le milieu génétique $\mathrm{MO}$, que la pénétrance est plus faible quand le mâle est un hétérozygote à phénotype non exprimé : $\mathrm{I} 2,9$ p. Ioo contre $66,0 \mathrm{p}$. Ioo quand le mâle est un hétérozygote polydactyle; la différence est hautement significative : $\chi_{2}^{2}=30,3$; Prob. < o,OOI. Ici encore, le mâle hétérozygote à phénotype non exprimé inhibe l'apparition de l'anomalie.

\section{Géniteurs et pénétrance minimum : mécanisme.}

Dans ce paragraphe, nous n'étudions que les deux milieux génétiques les mieux représentés : RAP et MO. Nous analyserons d'abord les résultats concernant le milieu RAP, puis ceux concernant le milieu MO et nous tenterons ensuite une comparaison des résultats obtenus.

Dans le milieu RAP, lors du croisement de deux sujets polydactyles, sans préciser leur degré d'expressivité (exp. III, tabl. II), nous trouvons une pénétrance de 1'ordre de 83,9 p. Ioo. Remplaçons un géniteur polydactyle par un mâle ou une femelle normale RAP. Ia pénétrance dans l'un ou l'autre cas n'est plus que de l'ordre de $5 \mathrm{I}$ p. IOo (50,3 p. Ioo quand le géniteur normal est une femelle - exp. $\mathrm{II}_{a}$, tabl. 7 et 52,4 p. roo quand le géniteur normal est un mâle-- exp. II $_{b}$, tabl. 7). Ceci montre que le croisement d'un géniteur normal avec un sujet polydactyle abaisse fortement la pénétrance de l'anomalie. Notons que le sexe du géniteur normal n'entre pas en 
jeu. Si l'on se souvient de la comparaison entre les pénétrances en $\mathrm{F}_{1}$ et en $\mathrm{F}_{b}$ (tabl. S), on voit que l'origine du géniteur normal n'est pas indifférente. (Eın effet, d'après ce que nous avons dit précédemment, il suffisait d'utiliser un géniteur nornal pour obtenir une pénétrance moins forte). Pour $F_{m}$, nous utilisons bien un géniteur normal, une femelle $\mathrm{F}_{1}$ issue de l'exp. II et nous constatons que la pénétrance reste très forte, de l'ordre de celle que l'on rencontrait lors des croisements de deux géniteurs polydactyles (respectivement 80,2 p. Ioo et $\$ 3,9$ p. I00). On observe donc une différence de pénétrance dans la descendance des femelles génétiquement normales issues du croisement II et dans celle des femelles normales de la lignée pure RAP. Pour expliquer une telle différence, on peut penser que la femelle $F_{\text {: normale }}$ issue de l'exp. II aurait accumulé des gènes modificateurs dont l'effet serait de favoriser l'expression du caractère. 'Tenons compte à présent du degré d'expressivité de l'anomalie chez les géniteurs polydactyles et considérons en premier lieu les croisements entre géniteurs à fort degré d'expressivité (tabl. 12). Nous constatons que la pénétrance, dans le cas de croisement entre sujets atteints de polydactylie bilatérale est très forte $(92,2 \mathrm{p}$. Ioo) ; elle n'est plus que de $63,6 \mathrm{p}$. Ioo si l'on remplace le mâle polydactyle bilatéral par un mâle normal (exp. $\mathrm{II}_{h}$, tabl. 1o). Elle n'est que de $5 \mathrm{I}, 8 \mathrm{p}$. Ioo quand on remplace une femelle polydactyle bilatérale par une femelle normale. Ici encore, mâles et femelles normaux abaissent la pénétrance et ceci de façon similaire. Enfin, lors des croisements de deux géniteurs à faible expressivité (polydactylie unilatérale gauche ou droite, tab1. I2), nous trouvons une pénétrance de 67,0 p. IOO, qui passe à 48 , I p. roo par remplacement d'une femelle atteinte par une femelle normale (exp. II" tabl. ro) et à 24,5 p. Ioo dans le croisement inverse (exp. II , tabl. Io). On retrouve encore le fait que la présence d'un géniteur normal abaisse la pénétrance de la polydactylie. Cependant, ici, on peut dire plus : la pénétrance est significativement moins forte quand on remplace un mâle atteint par un mâle normal.

Cette analyse permet de déterminer quels types de croisements il faut réaliser, si notre but est de réduire la fréquence d'apparition de l'anomalie chez les descendants. Si l'on s'en tient à des croisements entre géniteurs atteints, il y a intérêt à utiliser des sujets atteints de polydactylie unilatérale gauche ou droite; en effet, pour ces types de croisements, on a une pénétrance de 67,0 p. Ioo contre 92,2 p. IoO dans les croisements entre géniteurs atteints de polydactylie bilatérale. On pourrait expliquer cette différence par la présence de gènes modificateurs qui seraient en petit nombre chez les sujets atteints de polydactylie unilatérale gauche ou droite, et en plus grand nombre chez les sujets fortement atteints.

On voit donc que 1'on obtiendra la pénétrance la plus faible en croisant des géniteurs atteints de polydactylie unilatérale gauche ou droite avec des géniteurs normaux. Il reste à dire que ce minimum est atteint lors du croisement : ơ normal $\times$ \& polydactyle unilatérale gauche ou droite $(24,5 \mathrm{p}$. Ioo).

Qu'en est-il dans le milieu MO?

Lors du croisement entre sujets polydactyles, sans tenir compte de leur degré d'expressivité (exp. III, tabl. II), on observe une pénétrance de 50,0 p. Ioo. Le remplacement d'une femelle polydactyle par une femelle normale donne une pénétrance de $22,5 \mathrm{p}$. 100, tandis que le remplacement réciproque ne fournit qu'une pénétrance de I3, I p. IOo. La présence d'un sujet normal, dans un croisement avec un sujet 
polydactyle, abaisse la pénétrance et l'on peut préciser que la pénétrance est encore plus faible avec un mâle normal qu'avec une femelle normale (r3, I p. roo contre 22,5 p. IOO).

Peut-on utiliser n'importe quel géniteur normal? Esst-il, par exemple, indifférent d'utiliser une femelle MO ou une femelle $F_{1}$ normale issue de 1'exp. II : croisement de retour (tabl. 8). Il semble que l'on puisse le faire. En effet, il n'y a pas de différence significative entre les pénétrances en $\mathrm{F}_{\mathrm{I}}$ et $\mathrm{F}_{b}(22,5 \mathrm{p}$. Ioo et $3 \mathrm{I}, 7 \mathrm{p}$. Ioo).

Si l'on considère les croisements entre géniteurs à fort degré d'expressivité, on obtient une pénétrance de 47,0 contre 24,2 p. Ioo lors du remplacement d'une femelle gravement atteinte par une femelle normale et contre $9,3 \mathrm{p}$. Ioo dans le croisement réciproque. Comme précédemment, on met en évidence le rôle du géniteur normal, mais de plus, on peut préciser ici celui du mâle normal.

Il ressort de cette analyse que si l'on cherche à diminuer le plus possible la pénétrance, on a intérêt à utiliser des géniteurs à faible degré d'expressivité croisés avec des géniteurs normaux. Précisons en outre, que dans ce but, il est préférable d'utiliser des mâles normanx à des femelles normales.

Il est clair que ces deux analyses, la première relative au milieu RAP, la seconde au milieu MO, présentent des résultats très similaires. En particulier, dans les deux milieux, pour abaisser la pénétrance, on utilisera de préférence, des géniteurs à faible degré d'expressivité croisés avec des mâles normaux. Il semble que ce type de croisement soit plus efficace dans le milieu MO que dans le milieu RAP ; on arrive à des pénétrances respectives de $9,5 \mathrm{p}$. Ioo contre $24,5 \mathrm{p}$. Ioo; il est vrai que dans le milieu MO nous avions au début une pénétrance de l'ordre de $50 \mathrm{p}$. Ioo, bien plus faible que celle observée pour le milieu RAP, so p. Ioo.

Mis à part le type de croisement effectué, les deux milieux génétiques considérés ici, sont profondément différents : le milieu RAP donne en général une pénétrance plus forte que le milieu MO.

\section{Distribution des sexes.}

Nous avons jugé utile de vérifier si la répartition des sexes, entre les différentes classes de mutants, était conforme aux prévisions théoriques I : I. Dans ce but nous avons étudié la distribution des sexes dans les quatre milieux génétiques.

Parmi les descendants hémiméliques les mâles et les femelles sont dans les proportions I : I et ceci, quel que soit le milieu génétique considéré ; il en est de même pour la descendance d'aspect normal, qu'il s'agisse de sujets normal overlaps ou de sujets à phénotype et à constitution génotypique normale $(+/+)$. C'est seulement parmi les descendants hétérozygotes polydactyles appartenant au milieu génétique MO que 1'on note une prédominance des mâles : 56,6 p. Ioo contre 43,3 p. Ioo de femelles, la différence est significative $\left(\chi_{1}^{2}=7,20 ;\right.$ Prob. $<0$, or $)$.

\section{DISCUSSION}

Cette étude génétique sur l'hémimélie longitudinale a porté sur 88 or souris appartenant à quatre milieux génétiques différents : $336 \mathrm{I} \mathrm{RAP,} 368 \mathrm{~g} \mathrm{MO}, \mathrm{I}_{74} \mathrm{C}_{3} \mathrm{H}$ et $577 \mathrm{C}_{57} \mathrm{BL}$. 
L'étude génétique préliminaire avait montré que les souris hémiméliques se comportent comme des homozygotes récessifs ((he/he), les sujets polydactyles comme des hétérozygotes $(+/$ he) .

Pour chacun des milieux génétiques étudiés, nous avons effectué neuf à douze types de croisements groupés en quatre séries principales. Quel que soit le croisement effectué, nous obtenons des descendants d'apparence normale non prévus par la théorie ou en excédent. Nous avons donc analysé la constitution génotypique de ces individus d'apparence normale et nous avons montré qu'ils se comportent comme des hétérozygotes ( $+/$ he) à phénotype non exprimé : normal ovorlaps.

La présence de sujets normals overlaps a été détectée au cours de l'étude de nombreuses mutations, récessives ou dominantes, et ceci dans différentes espèces. Pour ne nous en tenir qu'aux souris, nous citerons parmi les mutations dominantes: REED (r937) dans l'étude de l'anomalie caudale tused (Fu) et CASPARI et DAvid (I940) parmi les souris kinky tail. Pour les mutations récessives, la présence de sujets normal overlaps a été signalée par HunT et PERMAR (I9I8) et HUN', MrX'TER et Permar (I937), lors de l'étude sur les souris flexed. LitTtle et BaGg (I923) lors de l'étude du phlyctène myencéphalique, par REEn (I936) parmi les sujets atteints de bec de lièvre, Koboziefr et Pomriaskinsky-KoboziefF (I940) dans la troncature de loreille, KoBOzIEFF, Dorllius et POMriaskinsky-KoboziefF (r955) dans la cataracte et KOBOZIEFF, POMRIASKINSKY-KOBOZIEFF et GEMÄHLing (Ig63) dans 1'hypotrichose périodique.

Notre mutation, hémimélie longitudinale (he), est génotypiquement semblable à celle décrite par CARTER (I95I), sous le nom de luxate (symbole $l x$ ) et par GREEN (I955) sous le nom de luxoid (symbole $l u$ ).

Pour étudier la pénétrance chez les hétérozygotes polydactyles, il était indispensable de montrer que la pénétrance des homozygotes était absolue. Ceci a été fait, après avoir groupé l'ensemble des sujets polydactyles et normal overlaps. Dans les croisements de sujets homozygotes hémiméliques avec des hétérozygotes à phénotype exprimé ou non, la répartition des homozygotes hémiméliques est dans les proportions I : I, tandis que dans les croisements entre hétérozygotes à phénotype exprimé ou non avec des homozygotes, la répartition des homozygotes est dans les proportions 3 : I.

CARTER (I95I) a, lui aussi, pour la mutation luxate constaté que la pénétrance est absolue pour les homozygotes, Green (I955) et Forsthoefei, (I958) ont fait la même constatation pour la mutation luxoid.

Ceci étant, nous avons étudié la pénétrance des hétérozygotes polydactyles; elle $n$ 'est pas absolue et soumise à différentes influences que nous avons cherché à mettre en évidence.

L'infuence du milieu génétique a été constatée quel que soit le type de croisement effectué. Dans la série de croisements entre ô normal et $q$ hémimélique, la pénétrance est forte dans les milieux $\mathrm{C}_{57} \mathrm{BL}$ et $\mathrm{RAP}$ sans qu'on puisse toutefois les distinguer (respectivement 48,4 p. Ioo et 36,6 p. I0o : $\chi_{1}^{2}=r, 47$ ) ; plus faible dans le milieu $\mathrm{C}_{3} \mathrm{H}$ ( I 9,4 p. IOO), et très faible dans le milieu MO $(2,5$ p. IOO). Cette situation se retrouve dans les résultats des croisements entre sujets polydactyles et normaux, du moins quand on ne prend pas en considération le degré d'expressivité de l'anomalie chez les sujets polydactyles. La figure r résume l'influence du milieu génétique dans ces deux séries de croisements. 
244 N. KOBOZIEFF, N. POMRIASKINSKY-KOBOZIEFF, E. GEMAHLING, F. REYNÈS

Dans la suite de cette étude de la pénétrance, nous n'avons pris en considération que les milieux génétiques les mieux représentés : RAP et MO.

La pénétrance est nettement plus élevée dans le milieu RAP que dans le milieu MO, à l'exception de la série de croisements entre ơ polydactyle et $q$ hémimélique où l'on ne note pas de différence statistique entre les pénétrances (respectivement I p. 100 et 66 p. I00: $\left.\chi_{i}^{2}=1,2\right)$.

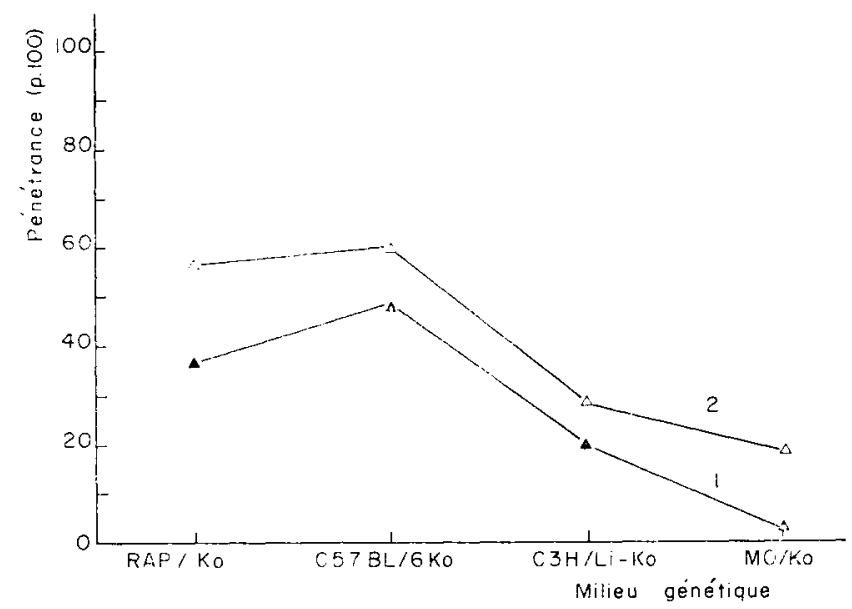

Fis. 1, - In/luence du milien sur la pénétrance

(I) normal $\times$ hémimélique

(2) normal $\times$ polydactyle

L'infuence des géniteurs polydactyles et normal overlaps sur la pénétrance, est apparue à la suite de croisements avec des femelles normales. Quel que soit le milieu génétique, la pénétrance est statistiquement plus faible quand l'un des géniteurs est hétérozygote à phénotype non exprimé normal overlaps (fig. 2).

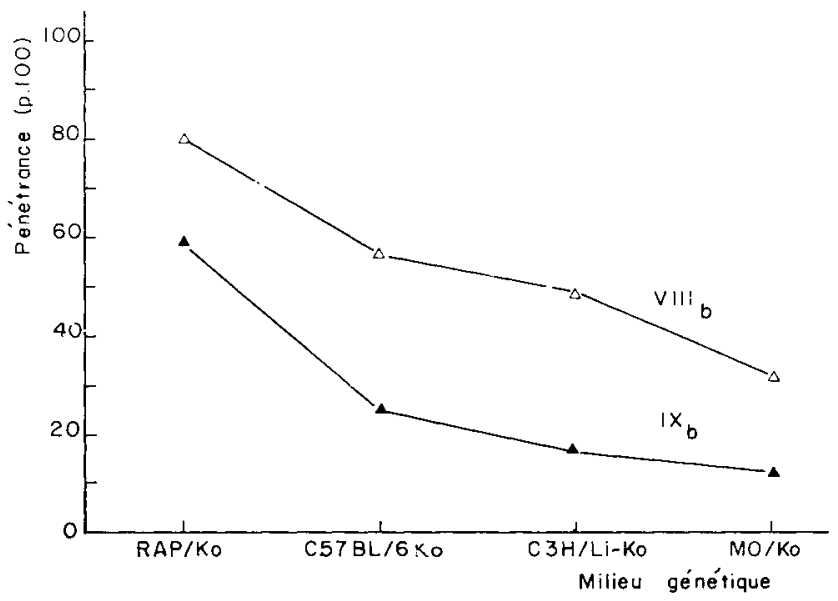

FIG. 2. - In/luence des géniteurs hétérosygotes sur la pénétrance (viII $\left.{ }_{b}\right)$ polydactyle $\times$ normal

$\left(\mathrm{IX}_{b}\right)$ normal ozerlaps $\times$ normal 
L'infuence du degré d'expression du phénotype des géniteurs sur la pénétrance elle aussi apparait clairement, aussi bien dans le milieu génétique RAP que dans le milieu MO : la pénétrance est forte lors du croisement de deux géniteurs à phénotype exprimé (polydactyles), plus faible quand le phénotype n'est exprimé que chez l'un d'entre eux (polydactule $x$ normal overlaps), et encore plus faible quand les deux géniteurs sont des hétérozygotes à phénotype non exprimé (normal overlaps $x$ normal overlaps) ; ceci quand on ne tient pas compte du degré d'expressivité de l'anomalie chez les géniteurs polydactyles (fig. 3). Quand on tient

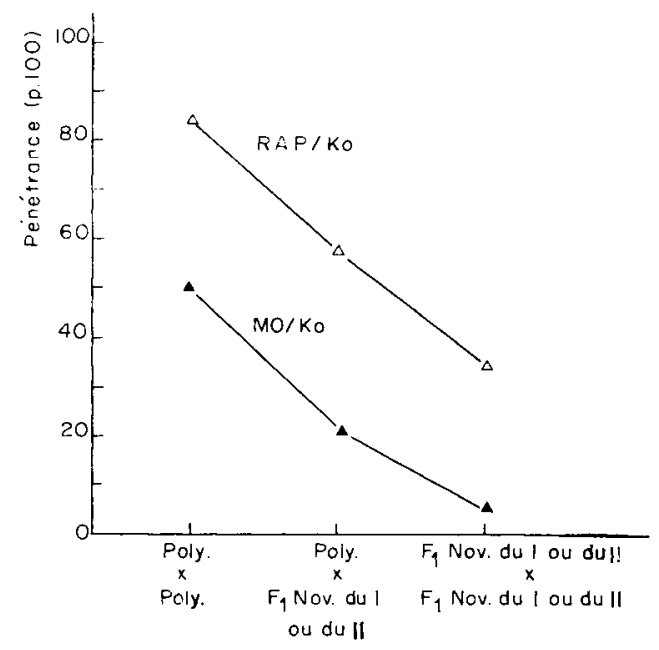

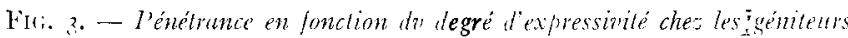

compte du degré d'expressivité de l'anomalie chez les géniteurs polydactyles, on constate que la pénétrance est plus forte dans la descendance de deux géniteurs à fort degré d'expressivité (polydactylie bilatérale), que dans celle de deux géniteurs à faible degré d'expressivité (polydactylie unilatérale gauche ou droite) ; ceci, pour le milieu génétique RAP. Dans ces mêmes types de croisements, on ne distingue pas de différence entre les pénétrances pour le milieu MO.

La pénétrance dans la première génération $\left(\mathrm{F}_{1}\right)$ est significativement plus faible dans les milieux génétiques $\mathrm{RAP}$ et $\mathrm{C}_{3} \mathrm{H}$, que dans la descendance issue ducroisement de retour $\left(\mathrm{F}_{b}\right)$, mais il n'y a pas de différence entre ces deux pénétrances pour les milieux $\mathrm{MO}$ et $\mathrm{C}_{57} \mathrm{BL}$. Le fait que la distribution des pénétrances en $\mathrm{F}_{1}$ ne soit pas identique à celles observées en $\mathrm{F}_{b}$ permet de penser à l'intervention de gènes modificateurs favorisant l'apparition de l'anomalie et dont l'accumulation serait rapide pour les milieux RAP et $\mathrm{C}_{3} \mathrm{H}$ et non décelable statistiquement au niveau des milieux $\mathrm{C}_{57} \mathrm{BL}$, et $\mathrm{MO}$ (fig. 4). L'un de nous avait réussi à augmenter la fréquence d'apparition de sujets anoures (KOBOZIEFF, I935), au cours d'une étude génétique sur l'anourie : un déficit notable des sujets anoures en $F_{1}$ avait été observé et nous avions obtenu une pénétrance absolue en $\mathrm{F}_{b}$.

L'infuence $d u$ sexe des géniteurs normaux a été détectée dans les croisenents entre sujets normaux et hétérozygotes polydactyles. Il a été possible dans ces croisements de distinguer cette influence, suivant que l'on considère ou non le degré 
d'expressivité de l'anomalie chez les géniteurs polydactyles. Sans tenir compte du degré d'expressivité de l'anomalie des géniteurs, nous avons montré que les géniteurs normaux mâles et femelles ont une action similaire sur la pénétrance dans les milieux $\mathrm{RAP}$ et $\mathrm{C}_{3} \mathrm{H}$, par contre, dans le milieu MO les mâles normau xabaissent

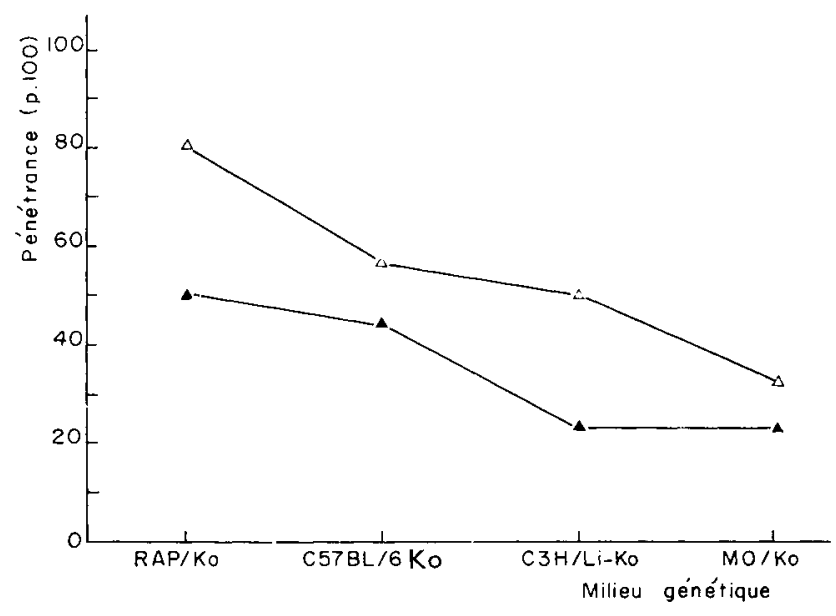

Fic. + - l'énétrance en lin et lil

davantage la fréquence d'apparition de la polydactylie que les femelles normales; enfin, dans le milieu $\mathrm{C}_{57} \mathrm{BI}$ ce sont les femelles normales qui ont cette influence Ce résultat est mis en évidence par la figure 5 .

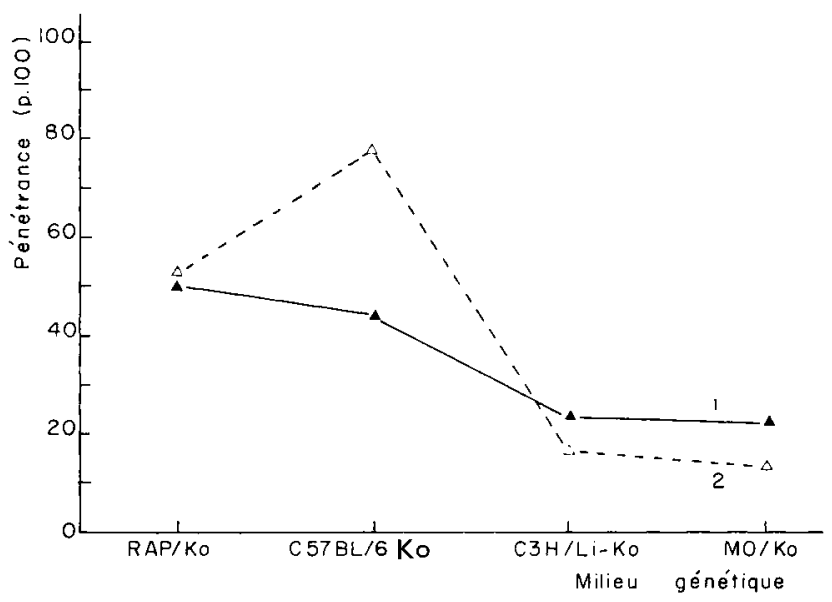

Fic. 5. - Influence du sexe des géniteurs normatux sur la pénétrance

(I) polydactyle $x$ normale

(2) normal $x$ polydactyle

Toujours dans le même type de croisement, si cette fois l'on tient compte du degré d'expressivité de l'anomalie chez les géniteurs du milieu génétique RAP, le mâle normal inhibe l'apparition de l'anomalie si la femelle est atteinte de polydac- 
tylie à faible expressivité (polydactylie gauche ou droite). Ceci est encore plus net dans le milieu génétique MO où l'on rencontrait déjà une différence significative entre les pénétrances relatives aux descendants issus des croisements entre mâle polydactyle et femelle normale et inversement.

REED (I937) en étudiant la mutation Fused $(F u)$, a signalé un phénomène semblable. Dans le croisement entre sujet normal et Fused, il obtient une pénétrance plus faible lorsque le mâle est normal.

Enfin, pour ce qui est de la distribution des sexes, à part un excédent de mâles polydactyles dans le milieu génétique MO, nous n'avons pas décelé de différence entre la proportion de mâles et de femelles ( $\mathrm{I}: \mathrm{I}$ ), quel que soit le milieu génétique et le type de croisement effectué.

\section{CONCLUSION}

Le gène he est semi-dominant : les homozygotes récessifs sont hémiméliques (he/he), tandis que les hétérozygotes $(+/$ he) sont à phénotype exprimé : polydactyles ou non : normal overlaps. De ce fait, la pénétrance n'est pas absolue à l'état hétérozygote, elle est très variable et dépend de différents facteurs :

- du milieu génétique : pénétrance forte dans le milieu RAP et $\mathrm{C}_{57} \mathrm{BL}$, faible dans le milieu $\mathrm{C}_{3} \mathrm{H}$ et très faible dans le milieu $\mathrm{MO}$;

- la pénétrance croît avec le degré d'expressivité de l'anomalie chez les géniteurs polydactyles ;

- du mode de croisement effectué qui permet d'abaisser la pénétrance; elle est plus faible en $F_{1}$ qu'en $F_{b}$ pour deux des milieux génétiques étudiés ;

- enfin, le sexe des géniteurs normaux utilisés et le degré d'expressivité de l'anomalie chez les géniteurs polydactyles ne sont pas indifférents : la pénétrance la plus faible a été observée dans la descendance issue du croisement ơ normal $\times$ o polvdactyle unilatérale gauche ou droite (milieux RAP et MO).

Recu pour publication en airil 1965.

\section{SUMMARY}

GENETICAL STUDY OF LONGITLDINAL HEMIMELIA IN MLCE PENETRANCE

For this study on longitudinal hemimelia we employed $880 \mathrm{I}$ mice, from four different backgrounds : $3361 \mathrm{RAP}, 3689 \mathrm{MO}$, I $174 \mathrm{C}_{3} \mathrm{II}$ and $577 \mathrm{C}_{57} \mathrm{BL}$.

For every one of these backgrounds we carried out four main series of crossing involving from nine to twelve types of experiences.

Hemimelic mice behave as recessive homozygotes of genetical constitution he/he, penetrancy is absolute.

In heterozygotes of genotypical constitution + /he, the phenotype is expressed or not (normal overlaps), the penetrancy is not absolute.

The penetrancy of polydactyly is variable in relation with the genetical background. We observe high frequency for RAP and $\mathrm{C}_{57} \mathrm{BL}$, lower for $\mathrm{C}_{3} \mathrm{H}$ and $\mathrm{MO}$ especially for the last one.

Besides we observed a variation of the freenuency within each genetical background : it depends on different factors :

- the degree of expressivity of the polydactyly of the begetters used in the crossing and

their sex;

- of the normal begetter;

- of the crossing pattern : penetrancy is lower in the first generation than in the offspring issued from backcross. 


\section{RÉFÉRENCES BIBLIOGRAPHIQUES}

Cister T.C., 1949. Genetic studies of luxale mice. Ph. D. Thesis Cambridge Vniv.

(Arter T. C., I95I. The position of fidget in linkage group) $V$ of the house mouse. J. Genel., 50. 26+-267

CASPARI J., Davin P. R., Igto. The inheritance of a tail abnormality in the house mouse. J. Hered., 31, $427-4,3$.

Forstroffel P. F., 195\%. The skeletal effects of the luxoid geme in the mouse, including its interactions with the luxate gene. 1. Morph., 102, 2-47-287.

GREeN H. C., 1955. A new hereditary leg and foot abnormality in the house nouse. J. Hered., 46, go-g9.

Grïnererc; 11., 1943. 'Two new mutant in the house mouse. I. Genet., 45, 1, 22-88.

Grúnererg H., 1047. Bilateral defect of the radius in a mouse. Acta Anat., 2, 270-274.

(iri:neber.; II., 1956. Genetical studies on the skeleton of the mouse. 'Three grenes for syndactylism. $J$. Genet, 54, I I 3-г 45 .

Hunt I. R., Mister R., Permar D., 1933. Flexed tail in the mouse Mlus musculus. Genetics, 18, 335366.

Hunt H. R., Pfrmar I), ig28. Filexed tail, a mutation in the house mouse. Inal. Rec., 41, 117.

Koвozime: X., 1935. Recherches morphologiques et génétiques sur l'auourie chez la souris. Thése de Doctorat ès Sciences. Bull. Biol. France ol Belgique, 69, $265-705$.

Koboziefr N., Dolifus M. A., Pondaskinsky-KoboziffF N. A., i955. Sur la rataracte, associce ou mon i d'autres malformations héréditaires chez la souris. ( $R$. Icud. Soi., 241, 1872-1874.

Koboziefi N., Pomrasisingy-Kobozielf N. A., i940. Sur les anomalies héréditaires de l'oreille exteme chez la snuris. C. R. Soc. Biol., 83, $386-388$.

Kobozifif N., Pomriaskinsks-KoboziefF N,, 1950. Sur l'hérédité des anomalies podales chez les souris $C . R, S$, dcad. Sci., 230, 579-5\% $\mathrm{I}$

KoboziefF N., Pomriaskivskr-Kobozlife X., i953. Recherches sur la constitution génotypique des souris luxées et polydactyles C. R. S. Soc. de Biol., 147, 196-199

Kobozieff N., Ponriaskinsk y-KobozierF N. A., i 95\%. Hémimćlie longitudinale chez la souris. I. Étude morphologique des hétérozyotes atteints de différentes anomalies du squelette du secoment distal. A. IlyperThalangie et polydactylic squelettique. Rec. Mél. Vél., 135, 877-goz.

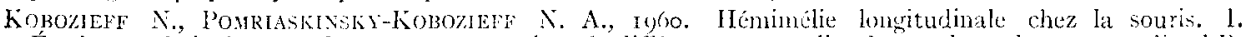
Etude morphologique des hétérozysotes atteints de différentes anonalies du squelette du segment distal B. Polyrlactylie intégrale. Rec. Méd. Vét., 136, 189-2 18.

KobozietF N., Pomriaskinsky-KoboziefF $Y$. A., ig61. Hémimélie longritudinale chiz la souris. 11. Étude morphologique des homozyotes atteints de différentes anomalies du squelette. A. Membres antérieurs. Rec. Méd. Vét., 137, 965-996.

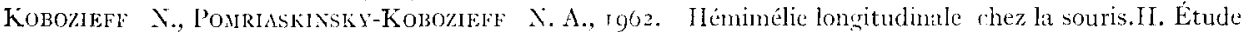
morphologique des homozygotes atteints de différentes anomalies du squelette. B. Membres postérieurs : hyperphalangie, oligodactylie et polydactylie squeletique. Rec. Méd. Vét., 138, 271-303.

Koboziefr N., Ponriasinsky-KonozmerF X. A., 1062. Iémimélic chez la souris. Il. Etudo morphologique des homozygrotes atteints de différentes anomalies du squelette. C. Membres postérieurs : polydactylie intégrale et cointure pelvienne. Rec. Méd. Vél., 138, 485-505.

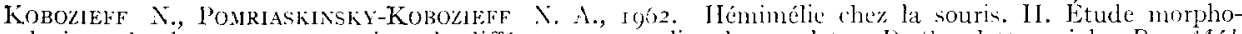
logique des homozygotes atteints de différentes anonalies du squelette. I). Squelette axial. Rec. Méd. Vél., 138, 67i-686.

Kobozieff N., Pomriaskinsky-Kobozikef X. A., Gemähling li., igbz. Iypotrichose périodique chez la souris. Ann. Biol. anim. Bioch. Biophys., 3, 207-218.

LiTTLE C. C., BAGG II., I g23. 'The occurence of two heritable types of abmormality amongr the desendants of X-rayed mice. J. Roenlgenol., 10, $975-989$.

REED S. C., I936. Harelip in the house mouse. I. Eiffects of internal and extemal environments. Genetics, 21, $339^{-} 3^{60}$.

REED S. C., I937. The inheritance and expression of fused, a new mutation in the house mouse. Genelics 22, $\mathrm{I}-13$.

SNEDECOR G. W., 1956. Stalistical methods applied to experiments in agricullure and biology. Iowa State Univ. Press, Ames, lowa, L. S. A., p. 534. 